\title{
A nonparametric kernel-based approach to Hammerstein system identification *
}

\author{
Riccardo S. Risuleo $^{\text {a }}$, Giulio Bottegal ${ }^{\text {a }}$, Håkan Hjalmarsson ${ }^{\text {a }}$ \\ ${ }^{a}$ ACCESS Linnaeus Centre, School of Electrical Engineering, KTH Royal Institute of Technology, Stockholm, Sweden
}

\begin{abstract}
Hammerstein systems are the series composition of a static nonlinear function and a linear dynamic system. In this work, we propose a nonparametric method for the identification of Hammerstein systems. We adopt a kernel-based approach to model the two components of the system. In particular, we model the nonlinear function and the impulse response of the linear block as Gaussian processes with suitable kernels. The kernels can be chosen to encode prior information about the nonlinear function and the system. Following the empirical Bayes approach, we estimate the posterior mean of the impulse response using estimates of the nonlinear function, of the hyperparameters, and of the noise variance. These estimates are found by maximizing the marginal likelihood of the data. This maximization problem is solved using an iterative scheme based on the expectation-conditional maximization, which is a variation of the standard expectation-maximization method for solving maximum-likelihood problems. We show the effectiveness of the proposed identification scheme in some simulation experiments.
\end{abstract}

Key words: System identification; Hammerstein systems; Nonlinear systems; Kernel-based methods; Gaussian processes;

\section{Introduction}

Nonlinear system identification is challenging because it is difficult to choose a general model structure to represent data from a nonlinear system $([47])$. One way to reduce the number of candidate models is to use blockoriented models; in this way, we can use model structures that are applicable to a wide array of problems and for which effective estimation techniques exist ([17]). Among the block-oriented models is the Hammerstein structure (HS). It is a nonlinear cascaded model where a linear time-invariant (LTI) dynamical model follows a static nonlinear mapping (see [27]).

The HS is capable of modeling a wide range of processes and has therefore been object of numerous studies $([25,51,3])$. Over the years, several identification methods have been proposed. In the following, we characterize the main approaches. In overparameterization methods, the problem of identifying the unknown parameters is embedded in the larger problem of identifying a vector containing all the cross products of the

\footnotetext{
* This paper was not presented at any IFAC meeting. Corresponding author R. S. Risuleo.

Email addresses: risuleo@kth.se (Riccardo S. Risuleo), bottegal@kth.se (Giulio Bottegal), hjalmars@kth.se (Håkan Hjalmarsson).
}

parameters $([2])$. This turns the problem into a linear identification problem, to which linear techniques can be applied (for instance, least squares in [2], instrumental variables in [23]). Once the overparameterized vector has been identified, the solution of the original problem is recovered by means of some reduction step (for instance, minimum norm in [2] and [23], consistent estimation in [9]). These methods hinge around a rankone constraint, which is difficult to enforce. Attempts have been made using regularization techniques (see, for instance, [42] and [16]). Subspace methods have been extended to HSs. The MOESP subspace method has been extended to HSs by assuming a polynomial model for the static nonlinearity ([49]). N4SID has been adapted to HSs by using support-vector machines ([18]). In Separable Least Squares methods, the unknowns are divided into two sets and the variables in one set are expressed as functions of the variables in the other set. This reduces the dimensionality of the problem ([51,19,24]). Similarly, in iterative methods, the variables are split into two sets, and the problem is solved by alternating the optimization between the two sets of variables (see, for instance, $[5,26])$. In stochastic methods the linearity is identified irrespective of the nonlinear transformation using correlation analysis $([7,39,21])$. These methods typically rely on certain properties of the input signal, for instance on its whiteness. Similarly, in blind methods, techniques from blind system identification are adapted 
to the identification of the linear component of the cascade $([4,48])$. The frequency-domain methods rely on the frequency content of the input and output signals. The output spectrum is a known function of the input frequency and magnitude, with some unknown parameters. By applying various sinusoidal inputs, these parameters can be identified (see, for instance, [6]). This requires that both the order of the polynomial input transformation and the order of the system are known. When these are unavailable, the harmonics of the output signal can be used to derive information about the nonlinear transformation in either a parametric or a nonparametric setting (see, for instance, [45,46,38]). In parametric-nonparametric methods, mixed descriptions are used for the components of the cascade (in a time-domain setting). For instance, the nonlinear transformation can be described with kernels or orthogonal series and the linear component with polynomial models (see $[22,20,31]$ ). An effective method based on maximum likelihood has been proposed in [52].

Besides a few exceptions (such as $[32,37,41]$ ), in most of the aforementioned works the authors consider parametric models for the LTI block of the HS, with known model order. Also, in many cases, the authors consider polynomial models for the nonlinear transformation, with known order. If the model structure and orders are unknown, which might be the case in many applications, we have to use complexity criteria such as AIC, BIC, or cross validation [27]; however, the choice of the best model is in general a very difficult problem.

In this paper, we extend the preliminary work presented in [41]. In that work, we considered HS with FIR models for the linear system and basis-function models for the static nonlinearity. The approach in [41] is, thus, limited to models where the noise is white and where the static nonlinearity is well represented by a model that is a linear combination of known basis functions. In this work, we relax the first modeling restriction by allowing for models with colored noise for the linear system (such as ARX, ARMAX, and Box-Jenkins models). To allow this relaxation, we use a nonparametric modeling approach where we model the impulse responses of the system in predictor form using Gaussian processes. We also relax the second modeling restriction by modeling the static nonlinearity with a Gaussian process.

To estimate the model, we follow an empirical Bayes ([28]) approach and we find an approximation of the posterior mean of the predictor impulse responses. This approximation depends on the parameters of the Gaussian-process model and on the noise variance (the hyperparameters), as well as on the input nonlinearity. To find these unknowns, we use a variation of the marginal-likelihood criterion. We maximize the likelihood of the data after integrating out the predictor impulse responses; the resulting problem is a joint maximum-a-posteriori/maximum likelihood prob- lem (JMAP-ML, see [53]). The proposed procedure is reminiscent of the marginal-likelihood approach for hyperparameter tuning in LTI system identification [33]; however, the presence of the static nonlinearity complicates the solution of the JMAP-ML problem, because of the large number of decision variables. To overcome this, we design a new iterative solution scheme based on an extension of the expectation-maximization (EM) method ([29]), known as expectation-conditional maximization (ECM, see [30]). In this way, we obtain a series of update rules for the estimates of the static nonlinearity and the hyperparameters. Except for one of the kernel hyperparameters, whose update is a scalar optimization over a finite domain, all the updates are available in closed-form.

It is worth stressing that Bayesian kernel-based methods using the stable spline kernel are not new in nonlinear system identification (see for instance $[32,37,41]$ ). The method in [37] is a fully nonparametric Bayesian method that can be used to identify any nonlinear model, without postulating specific structure. It works by identifying the model in nonlinear predictor form as a functional relationship between past inputs and the current output; however, it is not tuned specifically for the HS. Also the method in [32] is fully nonparametric, this time specific for the Wiener-Hammerstein model structure - that is, a static nonlinearity sandwiched between two linear timeinvariant models. In contrast with the present work it does not involve a marginalization step and thus cannot rely on the robustness properties of the empirical Bayes paradigm (see [50, Ch. 4], [33]).

The paper is organized as follows. In Section 2, we formulate the problem of system identification for the HS. In Section 3, we review the empirical Bayes approximation for the identification of the linear part of the system. In Section 4, we show the JMAP-ML criterion for the identification of the static nonlinearity and of the hyperparameters. In Section 5, we propose an ECM based algorithm to find the HS estimate. In Section 6, we compare, using simulations, our algorithm to standard system-identification tools for nonlinear system modeling. In Section 7, we give some concluding remarks. Appendix A contains the proofs of the main results.

\section{Problem formulation}

The HS we consider in this paper is the block-oriented nonlinear system structure given in Figure 1. It is the cascade composition of a static nonlinear function $f(\cdot)$ and a linear system $S$. In our formulation the system $S$ is a LTI discrete-time dynamic system (the linear system). We assume that the linear system is causal and exponentially stable. The linear system is fed by the input $\left\{w_{t}\right\}$ (unavailable to the experimenter), which is obtained by transforming the known input signal $\left\{u_{t}\right\}$ through the static nonlinear map $f(\cdot)$ (the static nonlinearity). 


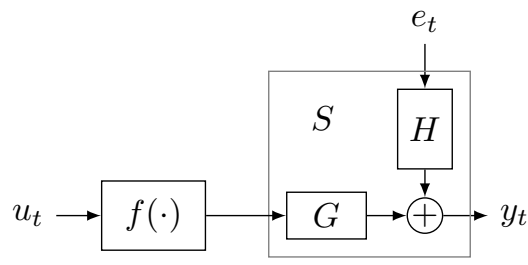

Figure 1. Block scheme of the HS studied in this paper.

The dynamics of the HS considered in this paper are described by

$$
\begin{aligned}
y_{t} & =\sum_{k=0}^{\infty} g_{k} w_{t-k}+\sum_{k=0}^{\infty} h_{k} e_{t-k}, \quad h_{0}=1, \\
w_{t} & =f\left(u_{t}\right),
\end{aligned}
$$

so that the linear system $S$ is a general Box-Jenkins model, where $\left\{g_{k}\right\}_{k=0}^{\infty}$ and $\left\{h_{k}\right\}_{k=0}^{\infty}$ are the impulse responses of the causal and exponentially stable filters $G$ and $H$ (with $H$ minimum phase), and $\left\{e_{t}\right\}$ zero-mean white Gaussian noise with unknown variance. We assume that the static nonlinearity is a uniformly bounded function.

In the classical prediction-error framework, we would introduce a parametric model for the system (see [27, Ch. 4] for a complete treatment), for example

$$
\begin{aligned}
y_{t} & =G_{\theta}(q) w_{t}+H_{\theta}(q) e_{t}, \\
w_{t} & =f_{\theta}\left(u_{t}\right),
\end{aligned}
$$

where $G_{\theta}(q)$ and $H_{\theta}(q)$ are strictly causal rational transfer functions ( $q$ represents the time-shift operator such that $\left.q^{-1} u_{t}=u_{t-1}\right)$ of opportune orders, and $f_{\theta}(\cdot)$ is a parametric model for the static nonlinearity. Using this model we would define the one-step ahead prediction of the output (supposing that $G_{\theta}(q)$ is stable and $H_{\theta}(q)$ is minimum phase and they are such that the predictor is stable),

$$
\hat{y}_{t}(\theta)=H_{\theta}^{-1}(q) G_{\theta}(q) f_{\theta}\left(u_{t}\right)+\left(1-H_{\theta}^{-1}(q)\right) y_{t},
$$

and minimize the squared prediction error to find the parameters $\theta$ :

$$
\hat{\theta}=\arg \min _{\theta} \sum_{t=1}^{N}\left\|y_{t}-\hat{y}_{t}(\theta)\right\|_{2}^{2} .
$$

In this work, following [34], we will start from a nonparametric model of the predictor

$$
\hat{y}_{t}=\sum_{k=1}^{\infty} a_{k} y_{t-k}+\sum_{k=0}^{\infty} b_{k} f\left(u_{t-k}\right),
$$

where we need to identify the impulse responses $\left\{a_{k}\right\}_{k=1}^{\infty}$ and $\left\{b_{k}\right\}_{k=1}^{\infty}$ and the static nonlinearity $f(\cdot)$. Comparing to (2), we want to identify the impulse responses of the filters $H_{\theta}^{-1}(q) G_{\theta}(q)$ and $1-H_{\theta}^{-1}(q)$, which we have supposed stable, without enforcing any particular parametric structure.

Remark 1 There are different approaches to recover the original transfer-function model from the estimated predictor. One approach is to truncate the impulse responses in (3) and consider the model as an autoregression. This allows us to say that $G(q) \approx \sum_{k=0}^{n_{b}-1} b_{k} q^{-k} /\left(1-\sum_{k=1}^{n_{a}} a_{k} q^{-k}\right)$ and $H(q) \approx$ $1 /\left(1-\sum_{k=1}^{n_{a}} a_{k} q^{-k}\right) ;$ to these high dimensional models we can now apply model-order reduction techniques (for more details, see [34]). Alternatively, we can use subspace methods to find a state-space representation of the process (similarly to what is presented in [13]). Furthermore, the nonparametric method gives no guarantees that the predictor impulse responses correspond to stable transfer functions in the simulation model (some methods to deal with this problem have been proposed in [44]).

The HS identification problem we consider is formulated as follows:

Problem 2 Given $N$ pairs of input-output samples $\left\{u_{t}, y_{t}\right\}_{t=1}^{N}$, collected from a system at rest (that is, $u_{t}=0, y_{t}=0$ for all $\left.t<0\right)$ reconstruct the predictor $i m$ pulse responses $\left\{a_{k}\right\}_{k=1}^{\infty}$ and $\left\{b_{k}\right\}_{k=0}^{\infty}$, and the nonlinear function $f(\cdot)$.

For sake of exposition and computation, we will consider the estimation of the first $n_{a}$ and $n_{b}$ samples of $\left\{a_{k}\right\}_{k=1}^{\infty}$ and $\left\{b_{k}\right\}_{k=0}^{\infty}$, respectively, with $n_{a}$ and $n_{b}$ large enough to capture the system dynamics:

$$
\hat{y}_{t}=\sum_{k=1}^{n_{a}} a_{k} y_{t-k}+\sum_{k=0}^{n_{b}-1} b_{k} f\left(u_{t-k}\right) .
$$

This truncation is not done to trade-off bias and variance but only for notational convenience, to be able to use the formalism of matrix algebra: $n_{a}$ and $n_{b}$ can be made arbitrarily large.

Remark 3 In this work, we present the identification procedure in the discrete-time domain. However, the same derivation can be carried out in the continuoustime domain, using the arguments in [35].

Remark 4 For notational convenience, we have assumed that the linear system is at rest when the identification experiment is performed. If the system is not at rest, there will be an effect from the unknown initial conditions that has to be accounted for. In [43], different methods are proposed to deal with unknown initial conditions in kernel-based system identification which can be adapted to the case of HS identification. 
Remark 5 The components of the HS can only be determined up to a scaling factor (see, for instance, [5]); in other words, for all $\alpha \in \mathbb{R}, \alpha \neq 0$, the pairs $\left(b_{t}, f(\cdot)\right)$ and $\left(\alpha b_{t}, \frac{1}{\alpha} f(\cdot)\right)$ describe the same input-output relation. To solve this indeterminacy, we constrain the kernel of the model of the static nonlinearity (see Section 4.1).

\subsection{Notation}

Given a sequence of scalars $\left\{a_{t}\right\}_{t=1}^{m}$, we denote by $a$ its vector representation $a^{T}=\left[a_{1} \cdots a_{m}\right]^{T} \in \mathbb{R}^{m}$. For a matrix $A$, the notation $[A]_{i, j}$ indicates the element of $A$ in position $i, j$. Given a vector $a \in \mathbb{R}^{m}$, we define by $\mathbf{T}_{N \times n}(a)$ the operator that returns the $N \times n$ Toeplitz matrix of whose elements are the entries of $a$ :

$$
\left[\mathbf{T}_{N \times n}(a)\right]_{i, j}= \begin{cases}a_{i-j+1} & \text { if } 1 \leq i-j+1 \leq m \\ 0 & \text { otherwise }\end{cases}
$$

Lower case letters indicate, in general, column vectors and, when there is no confusion, capital letters indicate their Toeplitz form; so, given $a \in \mathbb{R}^{m}$, we have that $A:=$ $\mathbf{T}_{N \times n}(a)$, where $N$ and $n$ are consistent with the rest of the formula. The symbol " $\otimes$ " denotes the standard Kronecker product of matrices.

\section{Kernel-based identification of LTI systems}

In this section, we review the modeling and identification approach for LTI systems introduced in [37] and adopted in the paper.

Under the assumption that the system can be represented by the predictor (4), we can write the relationship between the available data $\left\{y_{t}\right\}$ and the predictor impulse responses (for a fixed input sequence $\left\{w_{t}\right\}$ ) as

$$
y_{t}=\hat{y}_{t}+e_{t}=\sum_{k=1}^{n_{a}} a_{k} y_{t-k}+\sum_{k=0}^{n_{b}-1} b_{k} w_{t-k}+e_{t},
$$

where $\left\{e_{t}\right\}$ is the innovation process, consisting in Gaussian white noise with unknown variance $\sigma^{2}([1])$.

Equation (5) can equivalently be expressed in matrixvector notation as:

$$
y=\bar{Y} a+W b+e .
$$

where $a$ and $b$ are vectors of dimension $n_{a}$ and $n_{b}$, respectively, containing the impulse-response samples. The matrix $W \in \mathbb{R}^{N \times n_{b}}$ is the Toeplitz matrix constructed using the unavailable input $w=\left[f\left(u_{1}\right), \ldots, f\left(u_{N}\right)\right]^{T} \in$ $\mathbb{R}^{N}$. The matrix $\bar{Y} \in \mathbb{R}^{N \times n_{a}}$ is the Toeplitz matrix of the shifted output samples $\bar{y}=\left[\begin{array}{llll}0 & y_{1} & \ldots & y_{N-1}\end{array}\right]^{T}$. The vector $e$ is a Gaussian random vector with covariance matrix $\sigma^{2} I_{N}$ that represents the noise in the measurements. From (6), we can see $a$ and $b$ as solutions of a linear regression. In the next section we solve this regression using Gaussian processes ([40]).

\subsection{Modeling the predictor impulse responses}

By introducing Gaussian-process priors for $\left\{a_{k}\right\}_{k=1}^{n_{a}}$ and $\left\{b_{k}\right\}_{k=0}^{n_{b}-1}$, we can model the unknown predictor impulse responses as independent Gaussian vectors (see [34, Section 4]):

$$
a \sim \mathcal{N}\left(0, \lambda_{a} K_{\beta_{a}}\right), \quad b \sim \mathcal{N}\left(0, \lambda_{b} K_{\beta_{b}}\right) .
$$

The covariance matrices of these Gaussian distributions can be chosen, for instance, as first-order stable spline kernels [35]:

$$
\left[K_{\beta_{a}}\right]_{i, j}:=\beta_{a}^{\max (i, j)}, \quad\left[K_{\beta_{b}}\right]_{i, j}:=\beta_{b}^{\max (i, j)} .
$$

In these definitions, the hyperparameters $\beta_{a}$ and $\beta_{b}$ are scalars in the interval $[0,1)$ that govern the decay velocity of the realizations drawn from (7). The scalar hyperparameters $\lambda_{a}$ and $\lambda_{b}$ are positive scaling factors that account for the gains of $a$ and $b$. Realizations drawn from (7) are almost surely BIBO stable ([35]) and can, therefore, be used to represent the stable predictor filters in (2). Besides this property, in Section 5 we will show that the first-order stable spline kernel allows for a convenient implementation of the EM method for HS identification. The approach we propose in this work is however not limited to the stable-spline kernel and can be used with any kernel suitable for system identification purposes (for a reference, see $[12,11,15]$ ).

\subsection{Identification of the LTI system}

Using a Bayesian interpretation of (7), we are looking for the minimum mean-square-error estimators of the predictor impulse responses as the posterior means of $\left\{a_{k}\right\}_{k=1}^{n_{a}}$ and $\left\{b_{k}\right\}_{k=0}^{n_{b}-1}$ given the data ([1]):

$$
a^{\star}=\mathbb{E}[a \mid y], \quad b^{\star}=\mathbb{E}[b \mid y] .
$$

These estimators depend on the values of the hyperparameters and on the true value of the static nonlinearity; because we do not know these, we cannot calculate the minimum mean-square-error estimates. From a fully Bayesian perspective, we should introduce prior distributions for these unknowns and marginalize their influence on (9). However, this would lead to distributions that are not available in closed form and are not tractable. Instead, we can make an empirical Bayes approximation of these true estimates, where we consider the missing quantities $\rho$ and $w$ as deterministic parameters to be estimated. From the likelihood (6) and the 
prior distribution (7), we can write the posterior distribution of the impulse responses in closed form (see [8], Chapter 3.3) as

$$
p(a, b \mid y ; \hat{w}, \hat{\rho})=\mathcal{N}\left(\left[\begin{array}{l}
m_{a} \\
m_{b}
\end{array}\right],\left[\begin{array}{cc}
P_{a} & P_{a b} \\
P_{b a} & P_{b}
\end{array}\right]\right),
$$

where we have considered fixed values $\hat{\rho}=\left[\hat{\lambda}_{a}, \hat{\beta}_{a}, \hat{\lambda}_{b}, \hat{\beta}_{b}\right.$, $\left.\hat{\sigma}^{2}\right]$ for the hyperparameters and $\hat{w}$ for the input signal instead of the (unknown) true ones. The posterior means and covariances are given by

$$
\begin{aligned}
{\left[\begin{array}{cc}
P_{a} & P_{a b} \\
P_{b a} & P_{b}
\end{array}\right] } & =\left(\frac{1}{\hat{\sigma}^{2}}\left[\begin{array}{c}
\bar{Y}^{T} \\
\hat{W}^{T}
\end{array}\right]\left[\begin{array}{ll}
\bar{Y} & \hat{W}
\end{array}\right]+\left[\begin{array}{cc}
\left(\hat{\lambda}_{a} K_{\hat{\beta}_{a}}\right)^{-1} & 0 \\
0 & \left(\hat{\lambda}_{b} K_{\hat{\beta}_{b}}\right)^{-1}
\end{array}\right]\right)^{-1}, \\
{\left[\begin{array}{l}
m_{a} \\
m_{b}
\end{array}\right] } & =\frac{1}{\hat{\sigma}^{2}}\left[\begin{array}{c}
P_{a} P_{a b} \\
P_{b a} P_{b}
\end{array}\right]\left[\begin{array}{l}
\bar{Y}^{T} y \\
\hat{W}^{T} y
\end{array}\right],
\end{aligned}
$$

with appropriate dimensions for $P_{a}, P_{b}, P_{a b}, P_{b a}$. Using the empirical Bayes method, we approximate the optimal solutions in (9) as

$$
\hat{a}:=m_{a}, \quad \hat{b}:=m_{b}
$$

in other words, we are not taking the expectations in (9) with respect to the unavailable true posterior distribution of $\left\{a_{k}\right\}_{k=1}^{n_{a}}$ and $\left\{b_{k}\right\}_{k=0}^{n_{b}-1}$, but with respect to its empirical Bayes approximation $p(a, b \mid y ; \hat{w}, \hat{\rho})$.

In the classical empirical Bayes approach, all unknowns are considered as deterministic parameters, and their estimates $\hat{w}$ and $\hat{\rho}$ are found with the marginal-likelihood criterion

$$
\begin{aligned}
\hat{w}, \hat{\rho} & =\arg \max _{w, \rho} \log p(y ; w, \rho) \\
& =\arg \max _{w, \rho}-y^{T}\left(\Sigma_{y}(w, \rho)\right)^{-1} y-\log \operatorname{det}\left(\Sigma_{y}(w, \rho)\right)
\end{aligned}
$$

where $\Sigma_{y}(w, \rho)=\lambda_{a} \bar{Y} K_{\beta_{a}} \bar{Y}^{T}+\lambda_{b} W K_{\beta_{b}} W^{T}+\sigma^{2} I_{N}$, is the pseudo-covariance matrix of $y$ (see [34]).

The use of empirical Bayes estimates of impulse responses is not new $([34,37,12])$. The marginal-likelihood hyperparameter-tuning criterion is effective when used for LTI system identification purposes because of its robustness against noise $([36,33])$. In the case of HSs, however, the marginal-likelihood criterion (14) is doomed to fail because of the high number of variables in the problem (recall that $w$ has the same dimension as the data vector $y$ ). To overcome this, a possible solution is to parameterize $f(\cdot)$ and to express it as a linear combination of a low number of basis functions, as proposed in [41]. In this way though, the user is required to choose both the type of basis functions and the dimensionality of the parameterization of $f(\cdot)$.

To solve (14), without using parametrizations of the static nonlinearity, we introduce a nonparametric model for $f(\cdot)$ in the form of a Gaussian-process prior.

\section{Kernel-based Hammerstein system identifi- cation}

In this section, we describe the proposed procedure to HS identification. Our approach builds upon the marginallikelihood criterion (14) and incorporates a nonparametric model for the function $f(\cdot)$.

\subsection{Modeling the nonlinearity}

We model $f(\cdot)$ as a Gaussian process with a certain kernel function $M_{\eta}(\cdot, \cdot)$ parameterized by a vector $\eta$. The Gaussian-process prior means that any vector of values of $f(\cdot)$ has a Gaussian distribution. Therefore the unknown input samples $w$ have a joint distribution given by

$$
w \sim \mathcal{N}\left(0, M_{\eta, u u}\right),
$$

where the covariance matrix $M_{\eta, u u}$ is determined by the kernel function according to $\left[M_{\eta, u u}\right]_{i, j}:=M_{\eta}\left(u_{i}, u_{j}\right)$. Suitable choices for $M_{\eta}(\cdot, \cdot)$ are, for instance, the cubic splines kernel or RBF kernels (In our experiments we use the Gaussian kernel, see Section 6). Note that there is no scaling factor pre-multiplying the kernel, because of the indeterminacy described in Remark 5 .

Remark 6 The distribution in (15) does not in general apply to the HS in (1): for a given distribution of the input, the intermediate signal $\left\{w_{t}\right\}$ is, in general, not Gaussian. We stress that (15) is the distribution of $w$ induced by the Gaussian-process model for $f(\cdot)$ we adopt in this work and is valid only if $\left\{u_{t}\right\}$ is given.

\subsection{Joint estimation of the hyperparameters and the nonlinearity}

Having introduced a prior for $f(\cdot)$, and hence for $w$, we can modify (14) in order to account for the additional information given by (15). We consider the following estimator

$$
\hat{w}, \hat{\rho}=\arg \max _{w, \rho} p(y \mid w ; \rho) p\left(w ; \eta^{\star}\right)
$$

where $p(y \mid w ; \rho)$ is the Gaussian distribution of the data conditioned on the vector $w$, for a fixed value of the hyperparameter vector, and $p\left(w ; \eta^{\star}\right)$ is the Gaussian distribution (15) for some fixed value of the hyperparameter $\eta$. In the following, we drop the explicit dependency on $\eta$, assuming $\eta=\eta^{\star}$. How to tune this hyperparameter will be discussed in Section 5.3. 
Criterion (16) involves quantities that are of different nature: $w$ is stochastic and has a prior distribution, while $\rho$ is deterministic. This is an instance of a JMAP-ML estimator, as it provides the maximum-a-posteriori estimate of the stochastic unknowns and the maximum-likelihood estimate of the deterministic unknowns ([53]).

Having introduced a distribution for $w$, we could find the marginal distribution of $y$ and estimate $\rho$ maximizing

$$
p(y ; \rho)=\int p(y \mid w ; \rho) p(w) \mathrm{d} w,
$$

effectively obtaining the maximum-likelihood estimate of $\rho$. However, the integration is not possible in closed form and does not allow for efficient computation, whereas the maximization in (16) allows for an effective implementation (see Section 5). Furthermore, despite its bias, the JMAP-ML may outperform the maximumlikelihood criterion, especially when data records are short $([53])$.

In the following, we show that estimating $\left\{w_{t}\right\}$ (that is, the value of the static nonlinearity at the input locations $\left.\left\{u_{t}\right\}\right)$ is enough to obtain an estimate of the value of the static nonlinearity at any point $x$. To establish this result, we will use the following:

Lemma 7 Consider the HS (1), where $f(\cdot)$ is described by a stochastic process and $\left\{u_{t}\right\}$ is an arbitrary input signal. Fix $w_{t}=f\left(u_{t}\right)$ and let $x$ be a point such that $x \notin$ $\left\{u_{t}\right\}$; then, $f(x)$ and $\left\{y_{t}\right\}$ are conditionally independent given $w_{t}$.

PROOF. From (5), we have that $\left\{y_{t}\right\}$, is uniquely determined by $a, b,\left\{w_{t}\right\}$, and $\left\{e_{t}\right\}$. Therefore, $p(f(x) \mid y, a, b, w)=p(f(x) \mid e, a, b, w)$. Because $e$ is white noise, it is independent from $f(x)$ and we have that $p(f(x) \mid e, a, b, w)=p(f(x) \mid a, b, w)$. Integrating out $a$ and $b$ we get $p(f(x) \mid y, w)=p(f(x) \mid w)$, which proves the lemma.

Lemma 7 implies that the distribution of the output samples only depends on the values of the static nonlinearity at the input samples and not on the whole static nonlinearity. In turn, this means that if we are given $\left\{w_{t}\right\}$ the posterior distribution of any point $f(x)$ does not depend on the output samples but only on $\left\{w_{t}\right\}$. Therefore, any posterior estimate of a value $f(x)$ of the static nonlinearity is uniquely determined by $\left\{w_{t}\right\}$; this is formalized in the following proposition.

Proposition 8 Under the hypotheses of Lemma 7, the $M A P$ estimate of the function $f(\cdot)$ at any point $x \in \mathbb{R}$ is

$$
f_{\mathrm{MAP}}(x)=\arg \max _{f(x)} p(f(x) \mid w)
$$

and the minimum-mean-square error estimate is

$$
f_{\mathrm{MMSE}}(x)=\mathbb{E}[f(x) \mid w] .
$$

PROOF. Consider the posterior distribution of the point $f(x)$, for any $x \notin\left\{u_{t}\right\}$, given $y$ and $w$. In view of Lemma 7 we have that $p(f(x) \mid y, w)=p(f(x) \mid w)$. Maximizing with respect to $f(x),(17)$ follows. Taking the average, (18) follows.

Thanks to Proposition 8 , the estimate of $f(\cdot)$ at any point is available in closed form, as a function of $w$, when $f(\cdot)$ is a Gaussian process. From the Gaussian-process model (15), we have that any set of values of $f(\cdot)$ has a Gaussian distribution, with zero mean and covariance determined by the kernel function $M_{\eta}(\cdot, \cdot)$; so, any point $f(x)$ has a joint Gaussian distribution with $w$ given by:

$$
\left[\begin{array}{c}
w \\
f(x)
\end{array}\right] \sim \mathcal{N}\left(\left[\begin{array}{l}
0 \\
0
\end{array}\right],\left[\begin{array}{ll}
M_{\eta, u u} & M_{\eta, x u}^{T} \\
M_{\eta, x u} & M_{\eta, x x}
\end{array}\right]\right) .
$$

In this expression, $M_{\eta, x u} \in \mathbb{R}^{1 \times N}$ and $M_{\eta, x x} \in \mathbb{R}$ are matrices determined by the kernel function:

$$
\left[M_{\eta, x u}\right]_{j}=M_{\eta}\left(x, u_{j}\right), \quad M_{\eta, x x}=M_{\eta}(x, x) .
$$

Therefore, the posterior mean in (18) can be written in closed form (see [1, App. A] for details) as

$$
f_{\mathrm{MAP}}(x)=M_{\eta, x u} M_{\eta, u u}^{-1} w .
$$

Using the estimate of $w$ from (16), we obtain the MAP estimator

$$
\hat{f}(x)=M_{\eta, x u} M_{\eta, u u}^{-1} \hat{w} .
$$

The validity of this estimator is supported by the following result:

Proposition 9 Let $p(w, f(x))$ be the Gaussian distribution (19). Then, for any $x \in \mathbb{R}$, the JMAP-ML estimate

$$
f^{\star}(x), w^{\star}, \rho^{\star}=\arg \max _{f(x), w, \rho} p(y \mid w, f(x) ; \rho) p(f(x), w),
$$

is equivalent to the estimates (16) and (20).

PROOF. Consider the JMAP-ML estimate (21). Using the conditional independence of $y$ and $f(x)$ given $w$ (see Lemma 7) we obtain

$$
f^{\star}(x), w^{\star}, \rho^{\star}=\arg \max _{f(x), w, \rho} p(y \mid w ; \rho) p(f(x) \mid w) p(w),
$$


We can solve (22) with respect to $f(x)$, for fixed values of $w$ and $\rho$, finding

$$
f_{w}^{\star}(x)=\arg \max _{f(x)} p(y \mid w ; \rho) p(w, f(x))=\arg \max _{f(x)} p(f(x) \mid w) .
$$

Since $f(x)$ and $w$ are jointly Gaussian, the maximum is

$$
f_{w}^{\star}(x)=\mathbb{E}[f(x) \mid w]=M_{\eta, x u} M_{\eta, u u}^{-1} w .
$$

Plugging this solution into (21) we obtain

$$
w^{\star}, \rho^{\star}=\arg \max _{w, \rho} p(y \mid w ; \rho) p(w),
$$

because the value of $p(f(x) \mid w)$ is independent of $w$ when $f(x)=f_{w}^{\star}(x)$. Comparing (24) and (16) we see that $w^{\star}=\hat{w}$ and $\rho^{\star}=\hat{\rho}$. Plugging $w^{\star}$ into (23) we obtain $f^{\star}(x)=M_{\eta, x u} M_{\eta, u u}{ }^{-1} w^{\star}=M_{\eta, x u} M_{\eta, u u}{ }^{-1} \hat{w}=\hat{f}(x)$ which proves the result.

Therefore, solving (16) first and then using the MAP estimate (20), is equivalent to solving (21); in other words, the two-step procedure we propose gives the full MAP estimate of the nonlinear function (together with the ML estimate of the hyperparameters). In addition, the JMAP-ML estimate of $f(x)$, or alternatively its MAP estimate, is uniquely determined by the estimate of $f(\cdot)$ at the input locations $\left\{u_{t}\right\}$. Therefore, it is sufficient to estimate the vector $w$ from (16) to obtain an estimate of the whole static nonlinearity $f(\cdot)$. In the next section, we will show how the EM method can be used to derive a simple iterative method to solve (16).

\section{Iterative identification scheme using the EM method}

\subsection{A brief review of the EM method}

The criterion (16) requires the solution of a nonlinear high-dimensional problem, where the number of decision variables grows with the number of data points. To build an effective solution scheme, we use the Expectation/Conditional-Maximization (ECM) method ([30]), which is a generalization of the standard EM method. The idea behind the EM method is to obtain estimates of the cost function in (16) and maximize these approximate cost functions. To be more specific, suppose we are given a general maximum-likelihood problem

$$
\hat{\theta}=\arg \max _{\theta} p(y ; \theta) .
$$

We can augment the problem with some latent variables (also known as missing data) $z$, obtaining the equivalent problem in the joint distribution

$$
\hat{\theta}=\arg \max _{\theta} \int p(y, z ; \theta) \mathrm{d} z .
$$

Here, the point is that the joint distribution should be easier to evaluate than the original distribution. The EM method provides $\hat{\theta}$ by iterating between two steps, starting from an arbitrary initial point:

(E-step) Given an estimate $\hat{\theta}^{(k)}$ after the $k$ th iteration of the scheme, compute

$$
\mathcal{Q}\left(\theta, \hat{\theta}^{(k)}\right):=\mathbb{E}[\log p(y, z ; \theta)]
$$

where the expectation is taken with respect to the conditional distribution $p\left(z \mid y ; \hat{\theta}^{(k)}\right)$.

(M-step) Update the parameter estimate with

$$
\hat{\theta}^{(k+1)}=\arg \max _{\theta} \mathcal{Q}\left(\theta, \hat{\theta}^{(k)}\right) .
$$

When the M-step is difficult to solve, we can use ECM. In ECM, the maximization is split into a sequence of constrained optimization problems.

In HS case, we consider the complete-data loglikelihood - that is, the logarithm of the joint distribution of all the stochastic quantities - given by

$$
L(y, a, b, w ; \rho):=\log p(y, a, b, w ; \rho),
$$

with $a$ and $b$ acting as latent variables. In the E-step we calculate the function

$$
Q\left(\rho, w, \hat{\rho}^{(k)}, \hat{w}^{(k)}\right):=\mathbb{E}[L(y, a, b, w ; \rho)],
$$

where the expectation is taken with respect to the distribution (10), with $\hat{\rho}$ and $\hat{w}$ replaced by their current estimates $\hat{\rho}^{(k)}$ and $\hat{w}^{(k)}$. According to the ECM paradigm, we perform two conditional M-steps:

- Update $\hat{w}^{(k)}$ maximizing (26) with respect to $w$, keeping $\rho$ fixed to its previous value:

$$
\hat{w}^{(k+1)}=\arg \max _{w} Q\left(\hat{\rho}^{(k)}, w, \hat{\rho}^{(k)}, \hat{w}^{(k)}\right) .
$$

- Update $\hat{\rho}^{(k)}$ maximizing (26) with respect to $\rho$, keeping $w$ fixed at $\hat{w}^{(k+1)}$ :

$$
\hat{\rho}^{(k+1)}=\arg \max _{\rho} Q\left(\rho, \hat{w}^{(k+1)}, \hat{\rho}^{(k)}, \hat{w}^{(k)}\right) .
$$

Iterating (26) and the sequence of maximizations (27) and (28), we obtain a sequence of estimates of nondecreasing likelihood. The convergence properties of this sequence are discussed in Theorem 14.

\subsection{Applying the ECM method to HS identification}

We now show how to use the ECM method to solve our problem. Suppose that we have computed an estimate 
$\hat{\rho}^{(k)}$ of the hyperparameter vector and an estimate $\hat{w}^{(k)}$ of the static nonlinearity. Define $\hat{m}_{a}^{(k)}$ and $\hat{m}_{b}^{(k)}$ as the posterior mean values of $a$ and $b$ calculated by plugging $\hat{\rho}^{(k)}$ and $\hat{w}^{(k)}$ into (12); in addition, define $\hat{P}^{(k)}$ as the posterior covariance matrix computed by plugging the same parameter estimates into (11). Define the matrix

$$
\hat{S}^{(k)}=\left[\begin{array}{cc}
\hat{S}_{a}^{(k)} & \hat{S}_{a b}^{(k)} \\
\hat{S}_{b a}^{(k)} & \hat{S}_{b}^{(k)}
\end{array}\right]:=\hat{P}^{(k)}+\left[\begin{array}{c}
\hat{m}_{a}^{(k)} \\
\hat{m}_{b}^{(k)}
\end{array}\right]\left[\begin{array}{l}
\hat{m}_{a}^{(k)} \\
\hat{m}_{b}^{(k)}
\end{array}\right]^{T}
$$

which represents the second non-central moment of the estimate of the impulse responses. Furthermore, define the matrix $\mathbf{R} \in \mathbb{R}^{N n \times N}$ that satisfies the relationship $\mathbf{R} u=\operatorname{vec}(U)$, for all $u$.

Using these definitions, we now show that updating the estimate of the nonlinearity using the ECM method admits a closed-form expression.

Proposition 10 (Update of $\left.\hat{w}^{(k)}\right)$ Let $\hat{w}^{(k)}$ and $\hat{\rho}^{(k)}$ be estimates of the static nonlinearity and the hyperparameters at the kth iteration of the EM method. Define

$$
\begin{aligned}
\mathbf{A}^{(k)} & :=\mathbf{R}^{T}\left[\hat{S}_{b}^{(k)} \otimes I_{N}\right] \mathbf{R}+\hat{\sigma}^{2(k)} M_{\eta, u u}^{-1}, \\
\mathbf{b}^{(k) T} & :=y^{T} \mathbf{T}_{N \times N}\left(\hat{m}_{b}^{(k)}\right)-\operatorname{vec}\left(\bar{Y} \hat{S}_{a b}^{(k)}\right)^{T} \mathbf{R} .
\end{aligned}
$$

Then, the $(k+1)$ th estimate of $w$, as defined by $(27)$, is given by

$$
\hat{w}^{(k+1)}=\left(\mathbf{A}^{(k)}\right)^{-1} \mathbf{b}^{(k)}
$$

PROOF. See Appendix A.2.

The following result shows how to update the kernel hyperparameters of the linear system and the noise variance in the second conditional maximization step.

Proposition 11 (Update of $\left.\hat{\rho}^{(k)}\right)$ Let $\hat{w}^{(k+1)}$ be the update of the nonlinear transformation and $\hat{\rho}^{(k)}$ the estimate of the hyperparameters at the $k$ th iteration of the EM method. Define

$$
\begin{aligned}
\varphi_{a}\left(\beta_{a}\right) & =\operatorname{Tr}\left\{K_{\beta_{a}}^{-1} \hat{S}_{a}^{(k)}\right\} \\
Q_{a}\left(\beta_{a}\right) & =\log \operatorname{det} \beta_{a}+n_{a} \log \varphi_{a}\left(\beta_{a}\right), \\
\varphi_{b}\left(\beta_{b}\right) & =\operatorname{Tr}\left\{K_{\beta_{b}}^{-1} \hat{S}_{b}^{(k)}\right\} \\
Q_{b}\left(\beta_{b}\right) & =\log \operatorname{det} K_{\beta_{b}}+n_{b} \log \varphi_{b}\left(\beta_{b}\right) .
\end{aligned}
$$

Then, the $(k+1)$ th estimate of $\rho$, as defined by $(28)$, is given by

$$
\begin{aligned}
\hat{\beta}_{a}^{(k+1)} & =\arg \min _{\beta_{a} \in[0,1)} Q_{a}\left(\beta_{a}\right), \\
\hat{\lambda}_{a}^{(k+1)} & =\frac{1}{n_{a}} \varphi_{a}\left(\hat{\beta}_{a}^{(k+1)}\right) \\
\hat{\beta}_{b}^{(k+1)} & =\arg \min _{\beta_{b} \in[0,1)} Q_{b}\left(\beta_{b}\right), \\
\hat{\lambda}_{b}^{(k+1)} & =\frac{1}{n_{b}} \varphi_{b}\left(\hat{\beta}_{b}^{(k+1)}\right) \\
\hat{\sigma}^{2(k+1)} & =\frac{1}{N}\left(y^{T} y-\mathbf{b}^{(k) T} \hat{w}^{(k+1)}\right),
\end{aligned}
$$

where $\mathbf{b}^{(k)}$ is defined in (31).

PROOF. See Appendix A.3.

Remark 12 The update of $\sigma^{2}$ can also be written as

$$
\hat{\sigma}^{2(k+1)}=\frac{1}{N}\left(\|y-\mathbb{E}\{\hat{y} \mid y\}\|_{2}^{2}+\operatorname{Tr}\{\operatorname{Var}\{\hat{y} \mid y\}\}\right)
$$

where $\hat{y}=\bar{Y} a+\hat{W}^{(k+1)} b$, and the expectations are taken with respect to the posterior distribution (10). This shows that the estimated variance is, at each iteration, the sample prediction-error variance plus the variance of the predictor itself (given the current estimate of the static nonlinearity $\left.w=\hat{w}^{(k+1)}\right)$.

Using the factorization of the stable spline kernel, we can simplify the expressions in the previous proposition and provide more computationally attractive formulas. Let us introduce the $n$-dimensional discrete derivative operator

$$
\left[\Delta_{n}\right]_{i, j}= \begin{cases}1 & \text { if } i=j \\ -1 & \text { if } i=j-1 \\ 0 & \text { otherwise }\end{cases}
$$

and define the matrix $\Delta$ as the block diagonal matrix of $\Delta_{n_{a}}$ and $\Delta_{n_{b}}$. In this way, we introduce the noncentral second moment of the discrete derivative of the estimated impulse responses at iteration $k$ of the ECM method as

$$
\hat{D}^{(k)}:=\Delta \hat{S}^{(k)} \Delta^{T}
$$

Corollary 13 If $K_{\beta_{a}}$ and $K_{\beta_{b}}$ are given by first-order 
stable spline kernels (8), then

$$
\begin{aligned}
\varphi_{a}\left(\beta_{a}\right) & =\sum_{i=1}^{n_{a}-1} \frac{\hat{d}_{i}^{(k)}}{\beta_{a}^{i}-\beta_{a}^{i+1}}+\frac{\hat{d}_{n_{a}}^{(k)}}{\beta_{a}^{n_{a}}} \\
Q_{a}\left(\beta_{a}\right) & =\log \varphi_{a}\left(\beta_{a}\right)+\frac{n_{a}+1}{2} \log \beta_{a}+\frac{n_{a}-1}{n_{a}} \log \left(1-\beta_{a}\right) \\
\varphi_{b}\left(\beta_{b}\right) & =\sum_{i=1}^{n_{b}-1} \frac{\hat{d}_{n_{a}+i}^{(k)}}{\beta_{b}^{i}-\beta_{b}^{i+1}}+\frac{\hat{d}_{n_{a}+n_{b}}^{(k)}}{\beta_{b}^{n_{b}}} \\
Q_{b}\left(\beta_{b}\right) & =\log \varphi_{b}\left(\beta_{b}\right)+\frac{n_{b}+1}{2} \log \beta_{b}+\frac{n_{b}-1}{n_{b}} \log \left(1-\beta_{b}\right)
\end{aligned}
$$

where $\hat{d}_{i}^{(k)}:=\left[\hat{D}^{(k)}\right]_{i, i}$ is the ith element of the diagonal of $(38)$.

\section{PROOF. See Appendix A.4.}

The above results, combined together, show that the updates of the hyperparameter vector and the static nonlinearity can be decoupled and computed in closed-form. The only two parameters that require solving an optimization problem numerically are the kernel shaping parameters $\beta_{a}$ and $\beta_{b}$. However, as seen in Corollary 13, each of these parameters is updated by solving a scalar optimization problem in the interval $[0,1)$. Therefore, the whole estimation procedure can be implemented using only simple mathematical operations.

We are left with the question as to whether the sequence of updates converges. The following theorem provides the answer.

Theorem 14 Consider the JMAP-ML problem (16). For any value of the parameters $\hat{w}^{(k)}$ and $\hat{\rho}^{(k)}$, the updates $\hat{w}^{(k+1)}$ and $\hat{\rho}^{(k+1)}$ in Proposition 10 and Proposition 11 are such that

$$
\log p\left(y, \hat{w}^{(k+1)} ; \hat{\rho}^{(k+1)}, \eta^{\star}\right) \geq \log p\left(y, \hat{w}^{(k)} ; \hat{\rho}^{(k)}, \eta^{\star}\right)
$$

for all $k$. Furthermore, $\hat{w}^{(k)} \rightarrow w^{0}$ and $\hat{\rho}^{(k)} \rightarrow \rho^{0}$ as $k \rightarrow \infty$, where $w^{0}$ and $\rho^{0}$ are stationary points of the marginal likelihood $p\left(y, w ; \rho, \eta^{\star}\right)$.

PROOF. See Appendix A.5.

This theorem states that the sequences of updates for $\hat{w}^{(k+1)}$ and $\rho^{(k+1)}$, presented in Proposition 10 and Proposition 11, yield nondecreasing values of the JMAP-ML criterion. Furthermore, the sequences converge to stationary points of the JMAP-ML criterion.
These stationary points will, except for pathological cases, be local maxima of the marginal likelihoodeventual saddle points are numerically unstable, and minimal numerical perturbations will drive the iterates away from them ([29]).

The method thus provides a local solution to (16) - that is, it gives the estimates of the hyperparameter vector and of the static nonlinearity required in (9).

\subsection{Selecting the hyperparameter $\eta$}

The estimator (16) we propose depends on the choice of $\eta$. This can be seen as a model-selection problem. A standard way to deal with model selection is to use cross validation.

In this paper we propose the following marginal likelihood-type criterion:

$$
\eta^{\star}=\arg \max _{\eta} p(y, a, b ; \eta),
$$

where $a$ and $b$ are the true values of the predictor impulse responses. Notice that, from the conditional expectation formula, we have that $p(y, a, b ; \eta)=p(y \mid a, b ; \eta) p(a, b)$. Hence, we can consider the maximization of the likelihood only, with respect to the model $\eta$. Since $a$ and $b$ are not available, we replace them with their estimates $\hat{a}$ and $\hat{b}$ (which are functions of $\eta: \hat{a}=\hat{a}(\eta), \hat{b}=\hat{b}(\eta)$ ). We obtain the following criterion for model selection:

$$
\hat{\eta}=\arg \max _{\eta} \log p(y \mid \hat{a}(\eta), \hat{b}(\eta) ; \eta)
$$

To use this in practice, we would grid the values of $\eta$, estimating the impulse responses for each value of $\eta$ and choosing the $\eta$ that maximizes the criterion (39). Alternatively, we can integrate (39) into the ECM iterative scheme. At every iteration of the method we can calculate an estimate of the predictor impulse responses $\hat{a}^{(k)}$ and $\hat{b}^{(k)}$, found using the $k$ th value of the hyperparameters. Using these estimates we can update the model $\hat{\eta}^{(k)}$, with the maximization in equation (39):

$$
\hat{\eta}^{(k+1)}=\arg \max _{\eta} p\left(y \mid \hat{a}^{(k)}, \hat{b}^{(k)} ; \eta\right)
$$

In Section 6, we compare this estimation strategy with a cross-validation scheme, showing its effectiveness.

\subsection{HS identification algorithm}

The complete procedure for HS identification, coupled with the latter model selection criterion, is summarized in Algorithm 1. 


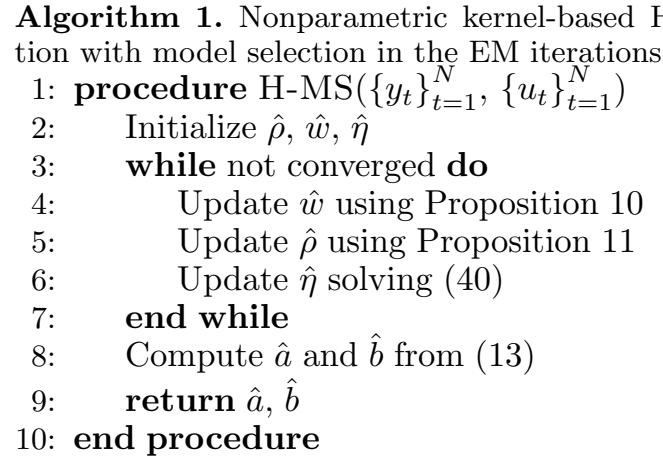

\section{Simulations}

To evaluate the performance of the proposed HS identification method, we present four Monte Carlo experiments. We want to assess the performance of the proposed identification scheme in different experimental setups. In the first, we identify HSs having ARMAX models as LTI blocks; we compare, for different identification dataset sizes, the proposed method for Hammerstein system identification with an oracle that knows the static nonlinearity. In the second, we identify HSs with OE models as LTI blocks. In the third, we evaluate the robustness of the method with respect to model misspecification. In the fourth, we evaluate the robustness of the method with respect to initializations.

Each experiment is made up of 500 Monte Carlo runs. At the $p$ th Monte Carlo run, we generate $N$ input-output pairs that are used to identify a model $\left(\hat{a}_{p}, \hat{b}_{p}, \hat{w}_{p}\right)$. Then, we generate a test set of 1000 new input-output pairs $\left\{u_{t, p}^{\text {test }}, y_{t, p}^{\text {test }}\right\}_{t=1}^{1000}$ and we calculate the output, $\left\{\hat{y}_{t, p}^{\text {test }}\right\}_{t=1}^{1000}$, and the transformed input, $\hat{w}_{p}^{\text {test }}$, predicted by the $p$ th model on the test set.

We evaluate the performance of the models using two indices (proposed in [34]) that quantify the generalization capability of the identified model on new data and the quality of the identified predictor impulse responses:

Coefficent of determination: At the $p$ th Monte Carlo run, we compute a measure of the fraction of output variance explained by the estimated predictor according to

$$
\mathrm{COD}_{p}:=1-\frac{\sum_{t=1}^{1000}\left(\hat{y}_{t, p}^{\mathrm{test}}-y_{t, p}^{\mathrm{test}}\right)^{2}}{\sum_{t=1}^{1000}\left(y_{t, p}^{\mathrm{test}}-\tilde{y}_{p}^{\mathrm{test}}\right)^{2}}
$$

where $\tilde{y}_{p}^{\text {test }}$ is the sample average of $y_{t, p}^{\text {test }}$.

Identification errors: At the $p$ th Monte Carlo run, we compare the predictor impulse responses of the identified models with the true ones. Similarly, we compare the identified static nonlinearity with the true one.
The quality of the identified model is quantified by

$$
\begin{aligned}
\operatorname{err}_{1, p} & =\frac{1}{2} \frac{\left\|\hat{a}_{p}-a_{p}\right\|_{2}}{\left\|a_{p}\right\|_{2}}+\frac{1}{2} \frac{\left\|\hat{b}_{p}-b_{p}\right\|_{2}}{\left\|b_{p}\right\|_{2}}, \\
\operatorname{err}_{2, p} & =\frac{\left\|\hat{w}_{p}^{\text {test }}-w_{p}^{\text {test }}\right\|_{2}}{\left\|w_{p}^{\text {test }}\right\|_{2}} .
\end{aligned}
$$

To compare the results of the different methods (in view of the general nonidentifiability of Hammerstein models, see Remark 5), we normalize all the identified system impulse responses such that they have unitary $\ell_{2}$ gain and positive first element.

\subsection{Experiment 1: Identification of HSs with ARMAX models}

In the first experiment, we generate 500 random Hammerstein models of the form

$$
y_{t}=\sum_{k=1}^{n_{y}} h_{i}^{y} y_{t-k}+\sum_{k=1}^{n_{u}} h_{i}^{u} f\left(u_{t-k}\right)+e_{t}+\sum_{k=1}^{n_{e}} h_{i}^{e} e_{t-k}
$$

where the coefficients of the ARMAX linearity are generated using the procedure described in [34]. First, we draw a value for $n_{y}$, uniformly in $\{1,2, \ldots, 20\}$. Then, we generate a random stable discrete-time $n_{y}$ th order system using the MATLAB function drmodel. The coefficients of the denominator of the transfer function of the model obtained provide the coefficients $\left\{h_{i}^{y}\right\}$. The number and values of the nonzero coefficients of the numerator define respectively $n_{u}$ and $\left\{h_{i}^{u}\right\}$ respectively. This ensures that the generated model has fixed input delay equal to one. Finally, we draw another model of the same order $n_{e}=n_{y}$ with drmodel and use the transfer function denominator coefficients to define $\left\{h_{i}^{e}\right\}$. The poles of the system and of the predictor are restricted to lie within a circle of radius 0.95 , and the output variance is bounded by 400 .

The static nonlinearity is the smooth and noninvertible Sinc function

$$
f(u)=\frac{\sin (6 \pi u)}{6 \pi u} .
$$

The system input is zero mean uniformly distributed white noise with variance $\operatorname{Var}\{u\}=1 / 3$.

In this experiment, we consider dataset sizes of $N=$ 250, 500, 1000 and we compare the following estimators:

Oracle The oracle estimator, which knows the static nonlinearity. It corresponds to the kernel estimator in (13) where the estimated input $\hat{w}$ is replaced by the true input $w$. The kernel hyperparameters and the noise variance are obtained maximizing the marginal likelihood of the output data, after integrating out the predictor impulse responses. 
H-MS (Hammerstein-Model Selection) The proposed nonparametric kernel-based HS estimator. It uses the ECM method to estimate the prior hyperparameters, the static nonlinearity, and the noise variance solving (16). The static nonlinearity is modeled using the Gaussian kernel

$$
\left[M_{\eta, u u}\right]_{i, j}=\exp \left[-\frac{\left(u_{i}-u_{j}\right)^{2}}{\eta}\right]
$$

This choice is motivated by the smoothness of the static nonlinearity. The parameter $\eta$ is tuned using the model-selection approach detailed in Section 5.3. The ECM iterations are stopped once the relative change between two iterations is below $10^{-2}$. We consider also the special case where we force $\beta_{a}=\beta_{b}$ to account for the ARMAX structure (as suggested in [34]).

H-CV (Hammerstein-Cross Validation) The proposed nonparametric kernel-based HS estimator, with a Gaussian-kernel model for the static nonlinearity and $\eta$ chosen through cross validation using 30\% of the training set as validation set. The $\eta$ is chosen in a grid of 50 values, logarithmically spaced between 0.01 and 10 .

Figure 2 shows the coefficients of determination of the models estimated in the identification experiment. In the left pane, we see the performance of the three methods when using the general predictor-identification method proposed in Algorithm 1. In the right pane, we see the performance of the three methods with $\beta_{a}=\beta_{b}$-as suggested in [34] for predictors of ARMAX-type linear systems.

In this simulation, the oracle represents the highest performance achievable by kernel-based methods with the stable-spline kernel-because it does not need to estimate the nonlinearity. From our results it seems that the proposed nonparametric method, which needs to estimate the full static nonlinearity together with the linear system, has a performance that is very close to the performance of the oracle that only needs to estimate the linear system.

From these simulations, it appears that the modelselection criterion (40) we have proposed compares favorably with the alternative cross-validation scheme. Furthermore, there is not a clear advantage in choosing $\beta_{a}=\beta_{b}$, even in the ARMAX case.

Figure 3 shows the identification errors for the linear part of the system $\left(\operatorname{err}_{1, p}\right)$ and for the nonlinear transformation $\left(\operatorname{err}_{2, p}\right)$ for the general model $\left(\beta_{a} \neq \beta_{b}\right)$. Observing these plots confirms the result: the proposed modelselection criterion (H-MS) has performance comparable to the cross-validation scheme. We omit the results for the ARMAX model $\left(\beta_{a}=\beta_{b}\right)$ because they are equivalent to those presented for the general model.
In general, the simulations confirm the effectiveness of the proposed nonparametric identification for HSs (assumed that a suitable prior is chosen; in this case the static nonlinearity is smooth so the Gaussian kernel is a valid choice). The simulation also confirms the validity of the proposed model-selection criterion.

\subsection{Experiment 2: Identification of HSs with OE models}

In this set of experiments, we generate rational OE models of the form

$$
y_{t}=\frac{N\left(q^{-1}\right)}{D\left(q^{-1}\right)} w_{t}+e_{t},
$$

where $N\left(q^{-1}\right)$ and $D\left(q^{-1}\right)$ are polynomials, in the discrete-time shift operator $q^{-1}$, of orders $n-1$ and $n$, respectively. The poles and zeros of the system are randomly sampled within the complex disk of radius 0.95 , uniformly in magnitude and phase. We run 5 sets of Monte Carlo experiments increasing the order of the LTI system, with orders $n=2,4,8,10,20$. Every experiment consists of 500 runs, and during every run we generate 1000 samples of input and output, with the output corrupted by Gaussian white noise with variance one tenth of the variance of the noiseless output. The input is uniform white noise of unit amplitude. The static nonlinearity is chosen as a seventh order polynomial whose coefficients are randomly generated at every run and are such that the roots of the polynomial are within the interval $[-2,2]$.

In this experiment we compare the following estimators:

NLHW The MATLAB function nlhw. It uses the prediction-error method to identify a parametric model of the system. To get the best performance from this method, we use the true orders of the LTI system and of the static nonlinearity and we initialize all the parameters in the optimization at their true values.

H-P (Hammerstein-Parametric) The semiparametric kernel-based HS estimator proposed in [41]. It estimates the samples of the static nonlinearity using a seventh-order polynomial model. The kernel hyperparameters and the noise variance are tuned with maximum marginal likelihood.

H-MS (Hammerstein-Model Selection) The proposed nonparametric kernel-based HS estimator with $n_{a}=0$. The static nonlinearity is modeled using the Gaussian kernel and the parameter $\eta$ is tuned using the proposed model-selection criterion.

H-CV (Hammerstein-Cross Validation) The proposed nonparametric kernel-based HS estimator with a Gaussian-kernel model for the static nonlinearity and $\eta$ chosen through cross validation using $30 \%$ of the training set as validation set. The $\eta$ is chosen in a grid of 50 values, logarithmically spaced between 0.01 and 10 . 

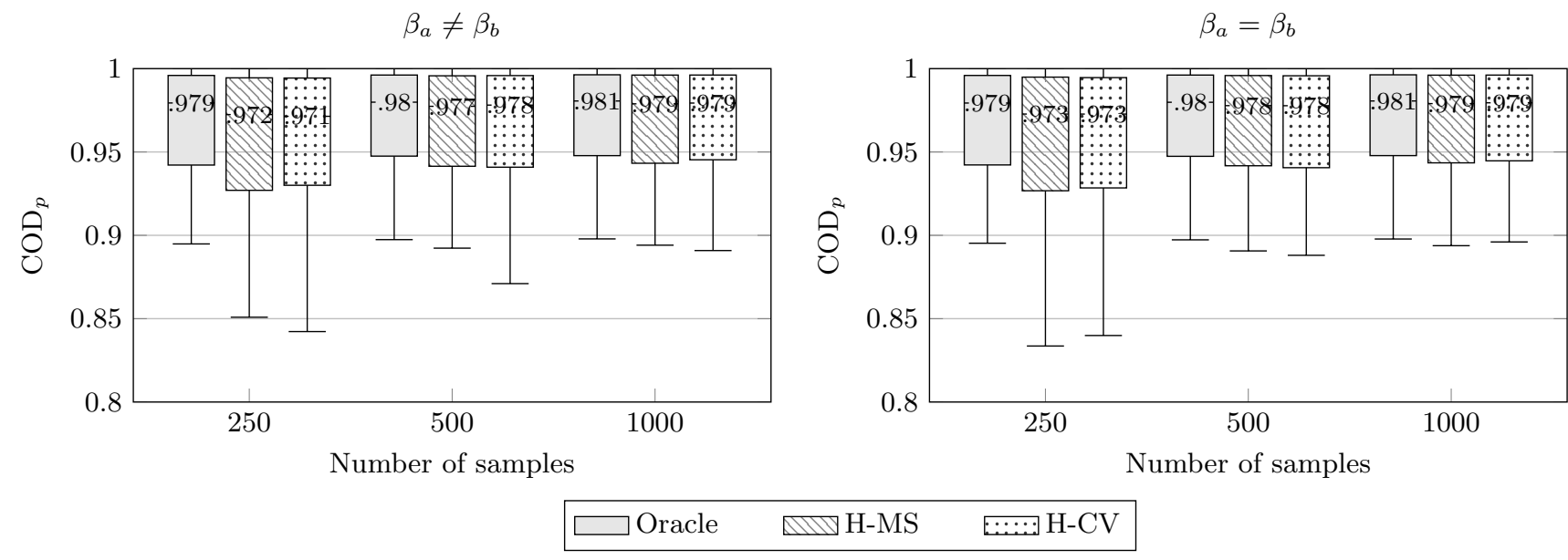

Figure 2. Experiment 1: Coefficients of determination on the test for Box-Jenkins (left pane) and ARMAX models (right pane) for different dataset sizes. The numbers in the boxplots indicate the median performance.

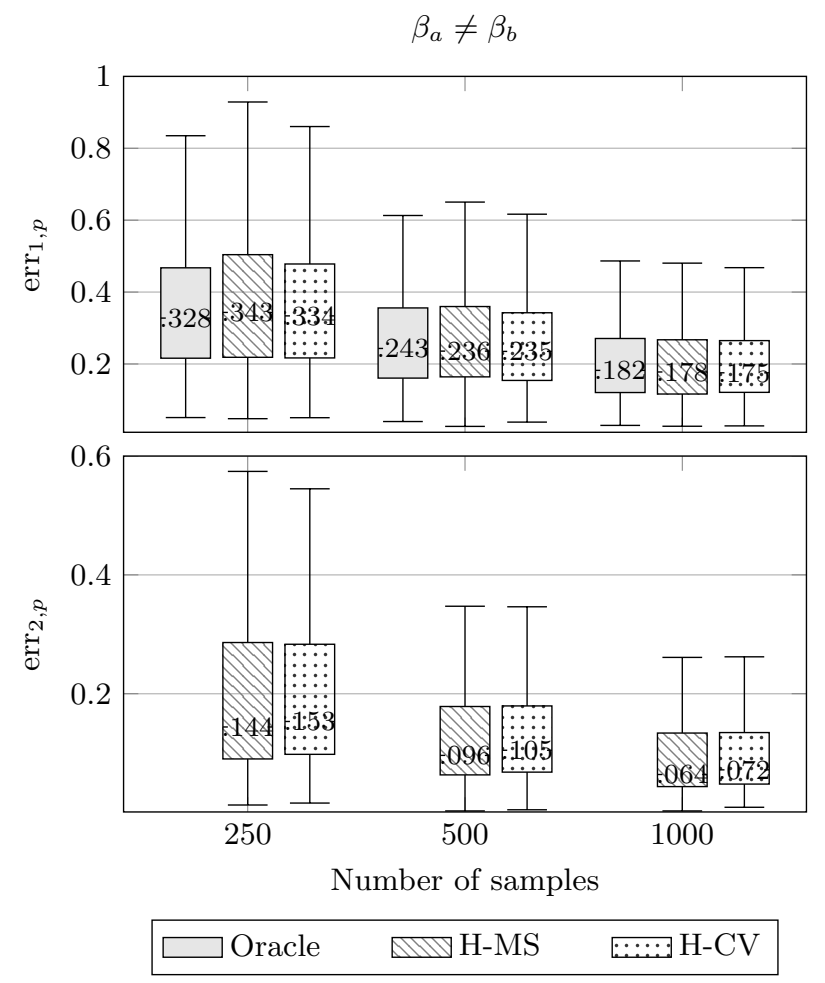

Figure 3. Experiment 1: Average model identification error for different dataset sizes. The numbers in the boxplots indicate median performance.

H-NR (Hammerstein-No Regularization) The proposed nonparametric kernel-based HS estimator without regularization for the estimate of the static nonlinearity. It estimates all samples of the static nonlinearity from (16), without using a prior for $w$ - that is, $p(w ; \eta)=1$.

H-QR (Hammerstein-Quadratic Regularization) The proposed nonparametric kernel-based HS esti- mator with quadratic regularization. It estimates all samples of the static nonlinearity from (16) using a standard-normal prior for $w$-that is, $M_{\eta, u u}=I_{N}$.

Figure 4 shows the coefficients of determination of the models estimated in the identification experiment for different orders of the unknown system. We do not plot the performances of $\mathrm{H}-\mathrm{QR}$ and $\mathrm{H}-\mathrm{NR}$ in this plot because they are much lower than the ones of the other methods (a look at Figure 5 shows why these methods have a poor generalization performance: adjacent samples of $w$ are modeled as independent; therefore, the methods have a hard time estimating $f(\cdot)$ for the input samples in the test set).

From Figure 4, it again seems that the model-selection criterion (40) compares favorably with the alternative cross-validation scheme. In the simulation, we have considered both parametric (NLHW) and nonparametric (H-P, H-MS, H-CV) models for the linear system. We note that the performance of the kernel-based methods for impulse-response identification is independent of the order of the estimated linear system whereas the performance of the parametric model decreases for larger orders. The slight degradation observed for H-P, H-MS and $\mathrm{H}-\mathrm{CV}$ for increasing model orders is due to the way the random LTI systems are generated: randomly sampling the higher-order models, we are more likely to encounter models with poles close to the unit circle or close to the negative real axis. In our experiments, we have observed that these kind of poles with high frequency content are difficult to identify using kernelbased methods with the first-order stable spline kernel (This was already observed in [34, Section 4.2]). The comparison of $\mathrm{H}-\mathrm{MS}$ and $\mathrm{H}-\mathrm{CV}$ made in ARMAX case remain valid here: the proposed model-selection method compares favourably with respect to the implemented cross-validation method. For low-order models, the performance of the prediction-error method implemented 


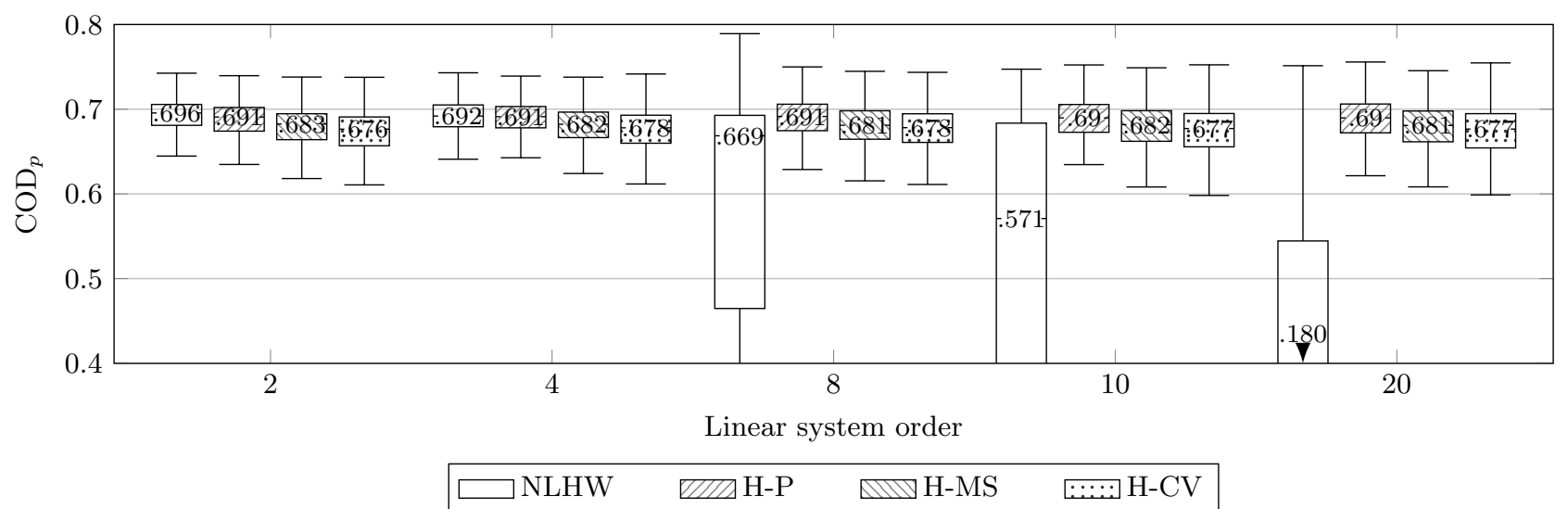

Figure 4. Experiment 2: Coefficients of determination on the test set for different orders of the linear system. The numbers in the boxplots indicate the median performance.

in nlhw is better than the nonparametric approach proposed; however, it degrades with the increasing model order. This degradation is possibly caused both by the increase in the variance of the estimates with the number of parameters to be estimated, as well as by the increasing complexity of the nonconvex optimization problems to be solved: in the last Monte Carlo experiment, the linear system of order 20 together with the static nonlinearity of order 7 give a total of 46 parameters to be estimated solving a nonconvex optimization problem.

Regarding the modeling of the static nonlinearity, the semiparametric method H-P has superior performance as compared to the proposed nonparametric methods; however, this depends on the correct choice of the type and order of the parametric model: with a fifth order model for the static nonlinearity, the median coefficient of determination of the models estimated by H-P drops from 0.69 to 0.51 (over the dataset of systems with $n=$ 8). Using a higher order has a less detrimental effect: with a ninth order model for the static nonlinearity, the median coefficient of determination of the models drops to 0.68 (over the dataset of systems with $n=8$ ). For models of higher order, the variance in the estimates causes the performance of the parametric method to drop further.

Our results indicate that the semiparametric method $\mathrm{H}-\mathrm{P}$ performs better than the proposed nonparametric method when the parametric model of the static nonlinearity is correctly specified (In the next section, we compare the methods when the used parametric model is not correct). In any case, using the nonparametric model we have the option to use any one of the many flexible kernels used for functional estimation and we do not have to rely on the correct specification of the basis functions of the nonlinearity.

The plots of the identification errors of the predictor impulse responses $\left(\operatorname{err}_{1, p}\right)$ and of the nonlinear transformation $\left(\operatorname{err}_{2, p}\right)$ are omitted in the interest of space.
The results remain valid: for low orders, NLHW performs better than the nonparametric methods; however, its performance degrades rapidly when the model order increases.
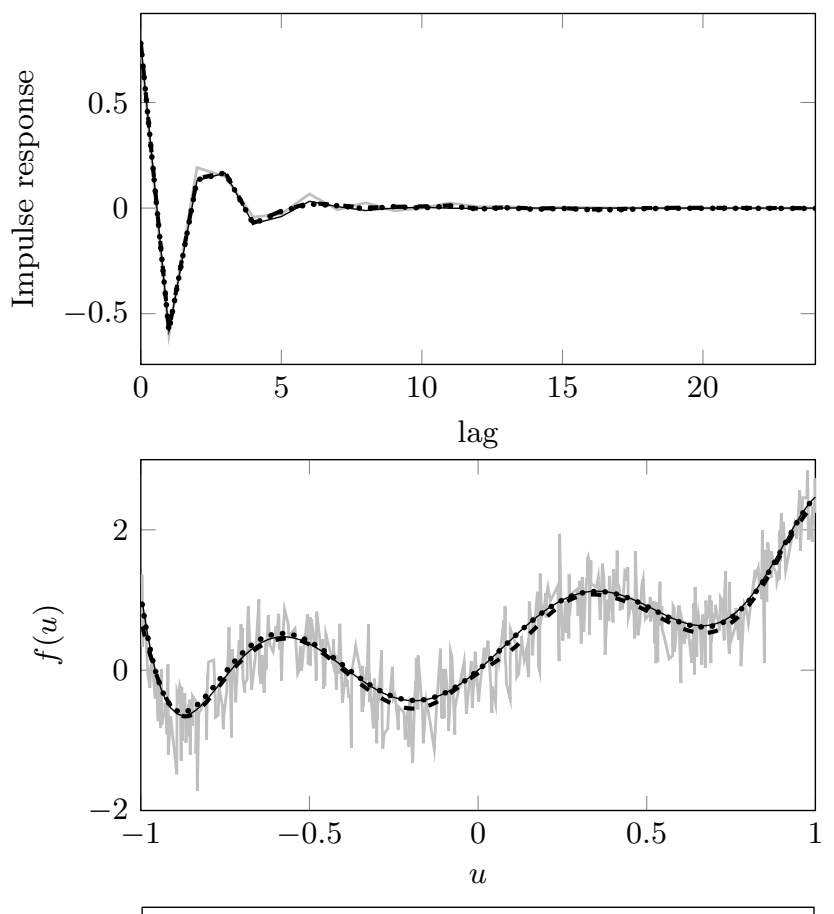

True $\cdots . . . \mathrm{H}-\mathrm{P} \quad-$ - H-MS $-\mathrm{H}-\mathrm{MF}$

Figure 5. Experiment 2: One realization of the identification experiment, we compare the performance of the parametric estimate H-P to the regularized estimate H-MS and to the nonregularized estimate $\mathrm{H}-\mathrm{NR}$ (The plot of $\mathrm{H}-\mathrm{QR}$ is indistinguishable from H-NR and is omitted).

Figure 5 shows a typical realization of the identification experiment. In this case, we can see that all methods are capable of recovering the impulse response of the linear system as well as the general shape of the static non- 
linearity. However, the lack of a regularizing term has a very detrimental effect on the estimated static nonlinearity (Note that the quadratic penalty in H-QR does not smooth the estimate; so, it has a performance that is similar to the nonregularized H-NR).

\subsection{Experiment 3: Robustness with respect to model misspecification}

In this section, we present a situation in which the nonparametric description of the nonlinearity used in H-MS gives an advantage over the parametric description used in H-P. We consider again 500 linear systems generated as presented in Section 6.2. In this case, the nonlinearity is given by the smooth and noninvertible function

$$
f(u)=\frac{\sin (20 u)}{20 u}
$$

We estimate the system using $\mathrm{H}-\mathrm{P}$ with different orders of the polynomial model of the static nonlinearity and we compare the performance of the estimated models (chosen with standard model-selection criteria) to the nonparametric model H-MS. In Figure 6, we present the median coefficient of determination of $\mathrm{H}-\mathrm{P}$ for different orders $n$ of the polynomial model. We also present the coefficients of determination of $\mathrm{H}-\mathrm{MS}$ and of $\mathrm{H}-\mathrm{P}$ with model order chosen with AIC, BIC, and cross validation (using $50 \%$ of the training data for validation).

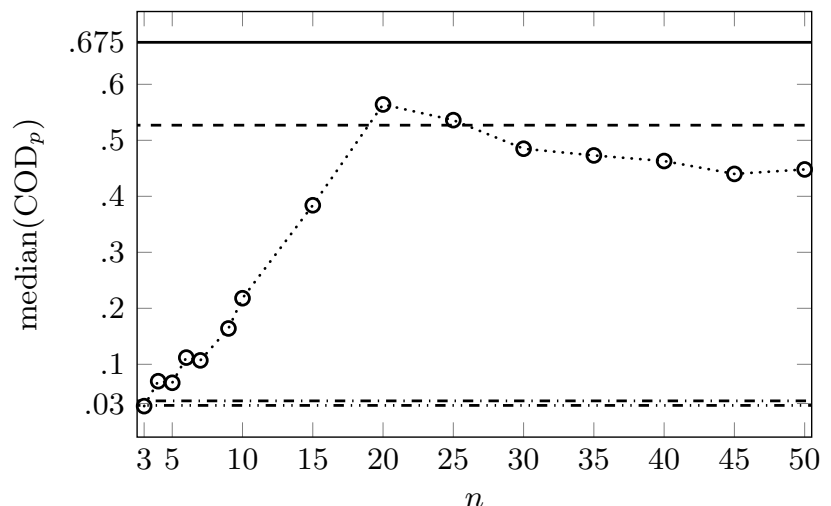

$\bullet \cdot \mathrm{H}-\mathrm{P} \quad-\cdots \mathrm{BIC} \quad-\cdots$-AIC $---\mathrm{CV} \quad-\mathrm{H}-\mathrm{MS}$

Figure 6. Experiment 3: Median coefficient of determination of the estimated semiparametric models (H-P) over the testing data for different orders $n$ of the polynomial model for the static nonlinearity. We also present the performance when using semiparametric models (H-P) when the model selection performed with $\mathrm{AIC}, \mathrm{BIC}$, cross validation (CV). We also present the result of the proposed nonparametric method (H-MS).

From this simulation, it seems that H-MS is able to model the nonlinearity effectively while $\mathrm{H}-\mathrm{P}$ suffers from the misspecification of the static nonlinearity. In particular, the parametric model required to capture the static nonlinearity is of very high order and this seems to trick the information-based model selection criteria into favoring models that suffer from severe undermodeling. The model selected with cross validation seems to perform better; however, to do standard cross validation we need to estimate one model for every order of the nonlinearity and compare their performance on the validation set. Because the estimation time of H-MS and H-P are comparable, running a full cross-validation scheme with 10 candidate models takes approximately 10 times longer than estimating one instance of H-MS.

\subsection{Experiment 4: Robustness with respect to initializa- tion}

To assess the robustness of the ECM method with respect to initialization of the algorithm, we have made one additional Monte Carlo experiment. In this experiment, we keep the training data fixed and restart Algorithm 1 from random initial conditions. We use the following initializations for the experiment:

$\operatorname{Exp~1:~} w_{t}^{(0)}=f\left(u_{t}\right)$ for $t=1, \ldots, N$, and $\eta=0.03$

Exp 2-500 $w^{(0)}$ drawn from the prior (41) with $\eta$ uniformly sampled among 12 values, logarithmically spaced between 0.01 and 10 .

In all cases, $\beta_{a}=\beta_{b}$ are sampled with uniform distribution in $[0.3,0.8], \lambda_{a}=\lambda_{b}$ are sampled with gamma distribution (shape parameter 5 , scale parameter 2 ), and $a$ and $b$ are started at their KB estimates (9). The noise variance $\sigma^{2}$ is initialized at the sample variance of the initial prediction error.

We compare the result of the identification of Exp 2-500 to the result of Exp 1. Figure 7 shows the histogram of the coefficients of determination of the estimates in the Exp 2-500. The figure also shows the performance of the estimate in $\operatorname{Exp} 1$.

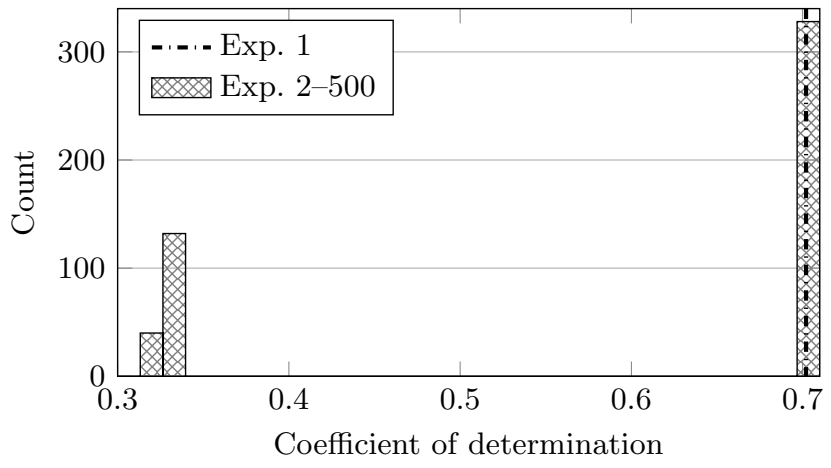

Figure 7. Experiment 4: Empirical distribution of the coefficients of determination of the identified models from randomly chosen initial conditions. The dashdotted line indicates the coefficient of determination of the model identified in $\operatorname{Exp} 1$. 
From this experiment, it appears that the method is robust to initialization. The method converges to a solution with a performance that is very close to the performance of the estimate initialized with the true static nonlinearity in a large number of cases (328 estimates out of 499 have a performance that is within 0.002 of the performance of the estimate in Exp 1). Considering that the estimates are initialized randomly and independently of the observed data, the method seems robust to initialization. The bimodal empirical distribution of the coefficients of determination in this experiment is given by the fact that, when $\eta$ is large, $w$ is allowed to be very irregular. In this case, the method can easily overfit the data by setting $\hat{w} \approx y, \hat{b} \approx \delta_{t}, \hat{a} \approx 0$, with opportune choices of the hyperparameters.

Given the performance with random initial conditions, it appears that the method could benefit very much from a good initialization procedure. One example could be to identify a parametric model of low order (see, for instance, [27]). Alternatively we could use the best linear approximation techniques (see $[46,38]$ ) to find an initial estimate of the linear system, and use its inverse to recover an estimate of the intermediate signal $\hat{w}$. Other initialization techniques for Hammerstein-Wiener identification have been proposed, that can be adapted to this application (see, for instance, [14] and references therein).

\section{Conclusions and future work}

In this paper, we have presented a kernel-based identification method for the identification of Hammerstein systems. We have modeled the LTI block and the static nonlinearity separately, using kernel-based approaches. The LTI block is modeled using the first-order stable spline kernel. The static nonlinearity is modeled using a kernel for function estimation. The static nonlinearity, the hyperparameters of the stable spline kernel, and the noise variance are estimated using a JMAP-ML criterion. The related optimization problem is solved by deriving an iterative method based on an EM-type algorithm, known as Expectation/Conditional-Maximization. The method is complemented with a model-selection criterion to tune the parameters of the nonparametric model of the input nonlinearity. The proposed method consists in a set of update rules that enable for a computationally simple identification procedure.

To evaluate the proposed method, we have performed a simulation study consisting of four scenarios. In the first, we have analyzed the ability of the method to estimate Hammerstein systems with a noninvertible nonlinearity and colored noise. We have compared the proposed method with an oracle that knows the nonlinear transformation and only needs to estimate the linear system. This oracle represents the upper bound on the performance achievable by kernel-based methods for this spe- cific problem. From this study, it appears that using a kernel-based model for the nonlinearity yields a performance that is close to the performance of the oracle. In addition, the proposed iterative model-selection criterion seems to be comparable with the alternative of using cross-validation for model selection. In the second scenario, we have compared the method with other parametric and semiparametric methods for HS identification. In this case, we have an optimal parametric model for the static nonlinearity; nonetheless, the performance of the proposed nonparametric method is surprisingly close to the performance of the semiparametric method. In the example we have presented, it even appears to perform better when the number of parameters in the polynomial model is incorrectly specified. In the third scenario, we have further compared the performance of the nonparametric model and the semiparametric model when the correct model of the static nonlinearity is not known; in particular, we have presented one situation in which the nonparametric model gives a clear advantage over a polynomial model. In the fourth scenario, we have shown the robustness of the EM-based method to the initialization.

We believe that the approach described in this paper has great versatility: it allows us to estimate HS where there is little information about the structure of the static nonlinearity. In addition, it may be employed for other system-identification problems involving uncertainties in the input, such as blind system identification or errorsin-variables system identification. These problems will be explored in forthcoming papers.

In this paper, we have moved the stochastic model for the static nonlinearity to the hyperparameter estimation level; effectively considering the model of the static nonlinearity as a hyperprior. An alternative approach is to consider the joint posterior distribution $p(a, b, w \mid y ; \rho)$, and find $\hat{a}, \hat{b}$, and $\hat{w}$ as the posterior means estimated with empirical Bayes. This approach has the problem that the joint posterior distribution is given by the convolution of the Gaussian random variables $w$ and $b$ and is, in general, not tractable. We are currently studying approximation methods for this posterior distribution using Markov Chain Monte Carlo and variational Bayes methods.

\section{Acknowledgment}

This work was supported by the Swedish Research Council via the projects NewLEADS (contract number: 201606079) and System identification: Unleashing the algorithms (contract number: 2015-05285).

\section{A Proofs}

In this section we report the proofs and explicit computations of the main results. 


\section{A.1 Computing $Q\left(w, \rho ; \hat{w}^{(k)}, \hat{\rho}^{(k)}\right)$}

In this subsection we derive the likelihood lower bound (26). Consider the complete likelihood:

$$
\begin{aligned}
& L(y, a, b, w ; \rho):=\log (p(y, a, b \mid w ; \rho) p(w)), \\
& =-\frac{1}{2 \sigma^{2}}\|y-\bar{Y} a-W b\|_{2}^{2}-\frac{N}{2} \log \left(\sigma^{2}\right) \\
& \quad-\frac{1}{2} w^{T} M_{\eta, u u}^{-1} w-\frac{1}{2} \log \operatorname{det}\left(M_{\eta, u u}\right) \\
& \quad-\frac{1}{2} a^{T}\left(\lambda_{a} K_{\beta_{a}}\right)^{-1} a-\frac{1}{2} \log \operatorname{det}\left(\lambda_{a} K_{\beta_{a}}\right) \\
& \quad-\frac{1}{2} b^{T}\left(\lambda_{b} K_{\beta_{b}}\right)^{-1} b-\frac{1}{2} \log \operatorname{det}\left(\lambda_{b} K_{\beta_{b}}\right) .
\end{aligned}
$$

If we take the expectation of $L$ with respect to the posterior density $p\left(a, b \mid y ; \hat{w}^{(k)}, \hat{\rho}^{(k)}\right)$, we can define the function

$$
\begin{aligned}
& Q\left(\rho, w ; \hat{\rho}^{(k)}, \hat{w}^{(k)}\right)=\mathbb{E}[L(y, a, b, w ; \rho)] \\
& =Q_{1}\left(w, \sigma^{2} ; \hat{w}^{(k)} ; \hat{\rho}^{(k)}\right)+Q_{2}\left(\lambda_{a}, \beta_{a} ; \hat{w}^{(k)}, \hat{\rho}^{(k)}\right) \\
& \quad+Q_{3}\left(\lambda_{b}, \beta_{b} ; \hat{w}^{(k)}, \hat{\rho}^{(k)}\right)+k
\end{aligned}
$$

where $k$ contains terms independent from the decision variables $w$ and $\rho$, and where

$$
\begin{aligned}
& Q_{1}\left(w, \sigma^{2} ; \hat{w}^{(k)}, \hat{\rho}^{(k)}\right)=-\frac{1}{2 \sigma^{2}}\left(-2 y^{T} W \hat{m}_{b}^{(k)}\right. \\
& \left.+\operatorname{Tr}\left\{W^{T} W \hat{S}_{b}^{(k)}+2 \bar{Y}^{T} W \hat{S}_{b a}^{(k)}\right\}+\sigma^{2} w^{T} M_{\eta, u u}^{-1} w\right),
\end{aligned}
$$

depends only on the decision variables $w$ and $\sigma^{2}$. Similarly, the functions

$$
\begin{aligned}
& Q_{2}\left(\lambda_{a}, \beta_{a} ; \hat{w}^{(k)}, \hat{\rho}^{(k)}\right)= \\
& \quad-\frac{1}{2} \operatorname{Tr}\left\{\left(\lambda_{a} K_{\beta_{a}}\right)^{-1} \hat{S}_{a}^{(k)}\right\}-\frac{1}{2} \log \operatorname{det}\left(\lambda_{a} K_{\beta_{a}}\right), \\
& Q_{3}\left(\lambda_{b}, \beta_{b} ; \hat{w}^{(k)}, \hat{\rho}^{(k)}\right)= \\
& \quad-\frac{1}{2} \operatorname{Tr}\left\{\left(\lambda_{b} K_{\beta_{b}}\right)^{-1} \hat{S}_{b}^{(k)}\right\}-\frac{1}{2} \log \operatorname{det}\left(\lambda_{b} K_{\beta_{b}}\right),
\end{aligned}
$$

depend only on the kernel hyperparameters. In the previous expressions, $\hat{S}_{a}^{(k)}, \hat{S}_{b}^{(k)}$, and $\hat{S}_{b a}^{(k)}$ are partitions of appropriate size of $\hat{S}^{(k)}$ - see $(29)$.

\section{A.2 Proof of Proposition 10}

Consider the maximization $Q\left(w, \rho, \hat{w}^{(k)}, \hat{\rho}^{(k)}\right)$ with respect to $w$. Using the decomposition in (A.1), we see that it is equivalent to the maximization of (A.2) with respect to $w$. That is to say

$$
\hat{w}^{(k+1)}=\arg \max _{w} Q_{1}\left(w, \rho^{2} ; \hat{w}^{(k)}, \hat{\rho}^{(k)}\right) .
$$

Observe that

$$
\begin{aligned}
\operatorname{Tr}\left\{W^{T} W \hat{S}_{b}^{(k)}\right\} & =w^{T} \mathbf{R}^{T}\left[\hat{S}_{b}^{(k)} \otimes I_{N}\right] \mathbf{R} w, \\
\operatorname{Tr}\left\{\bar{Y}^{T} W \hat{S}_{b a}^{(k)}\right\} & =\operatorname{vec}\left(\bar{Y} \hat{S}_{a b}^{(k)}\right) \mathbf{R} w, \\
W \hat{m}_{b}^{(k)} & =\mathbf{T}_{N \times N}\left(\hat{m}_{b}^{(k)}\right) w .
\end{aligned}
$$

Therefore we have:

$$
Q_{1}\left(w, \sigma^{2} ; \hat{w}^{(k)}, \hat{\rho}^{(k)}\right)=-\frac{1}{2 \sigma^{2}} w^{T} \mathbf{A}^{(k)} w+\frac{1}{\sigma^{2}} w^{T} \mathbf{b}^{(k)}
$$

with $\mathbf{A}^{(k)}$ and $\mathbf{b}^{(k)}$ defined in (30) and (31). So (A.4) has the closed form solution (32).

\section{A.3 Proof of Proposition 11}

Consider the maximization of (A.1) with respect to $\lambda_{a}$ and $\beta_{a}$. It corresponds to the maximization of (A.3). We first notice that

$$
\begin{aligned}
& Q_{2}\left(\lambda_{a}, \beta_{a} ; \hat{w}^{(k)}, \hat{\rho}^{(k)}\right)= \\
& \quad-\frac{1}{2 \lambda_{a}} \operatorname{Tr}\left\{K_{\beta_{a}}^{-1} \hat{S}_{a}^{(k)}\right\}-\frac{1}{2} \log \operatorname{det}\left(K_{\beta_{a}}\right)-\frac{n_{a}}{2} \log \left(\lambda_{a}\right) .
\end{aligned}
$$

For a fixed $\beta_{a}$, the maximum with respect to $\lambda_{a}$ is given by $\lambda_{a}^{\star}\left(\beta_{a}\right)=\operatorname{Tr}\left\{K_{\beta_{a}}^{-1} \hat{S}_{a}^{(k)}\right\} / n_{a}$. Plugging $\lambda_{a}^{\star}$ back in the expression for $Q_{2}$, we have a cost function of $\beta_{a}$ alone, which we can minimize with a grid search over $[0,1)$ :

$$
Q_{a}\left(\beta_{a}\right)=n_{a} \log \left(\varphi_{a}\left(\beta_{a}\right)\right)+\frac{1}{2} \log \operatorname{det}\left(K_{\beta_{a}}\right)
$$

where we have defined $\varphi_{a}\left(\beta_{a}\right)=\operatorname{Tr}\left\{K_{\beta_{a}}^{-1} \hat{S}_{a}^{(k)}\right\}$

Once we have the optimal $\beta_{a}$ for iteration $k$, we can calculate the corresponding optimal $\lambda_{a}$, obtaining equation (33).

With exactly the same procedure we can calculate the optimal $\lambda_{a}$ as a function of $\beta_{b}$, obtaining equations (35) and (34).

The update for $\sigma^{2}$ is found isolating in (A.1) the part

$$
-\frac{1}{2 \sigma^{2}} y^{T} y+Q_{1}\left(\hat{w}^{(k+1)}, \sigma^{2} ; \hat{w}^{(k)}, \hat{\rho}^{(k)}\right)-\frac{N}{2} \log \left(\sigma^{2}\right),
$$

which we need to maximize with respect to $\sigma^{2}$. First we notice, from (A.5), that

$$
Q_{1}\left(\hat{w}^{(k+1)}, \sigma^{2} ; \hat{w}^{(k)}, \hat{\rho}^{(k)}\right)=\frac{1}{2 \sigma^{2}} \hat{\mathbf{b}}^{(k) T} \hat{w}^{(k+1)},
$$

so optimizing (A.6) with respect to $\sigma^{2}$ yields (36). 


\section{A.4 Proof of Corollary 13}

To prove the result, we need the following lemmas.

Lemma 15 Consider the $n$-dimensional first-order stable spline kernel $K_{\beta}$; then,

$$
\log \operatorname{det}\left(K_{\beta}\right)=\frac{n^{2}+n}{2} \log (\beta)+(n-1) \log (1-\beta) .
$$

PROOF. For a fixed $\beta$, the first-order stable spline kernel admits the factorization ([10])

$$
K_{\beta}=\Delta_{n}^{-1} W_{\beta} \Delta_{n}^{-T},
$$

where $\Delta_{n}$ is defined in (37) and where

$$
W_{\beta}:=\operatorname{diag}\left\{\beta-\beta^{2}, \beta^{2}-\beta^{3}, \ldots, \beta^{n-1}-\beta^{n}, \beta^{n}\right\} \text {. }
$$

Therefore

$$
\begin{aligned}
& \log \operatorname{det}\left(K_{\beta}\right)=\log \operatorname{det}\left(\Delta_{n}^{-1} W_{\beta} \Delta_{n}^{-T}\right)= \\
& =\log \operatorname{det}\left(W_{\beta}\right)=\sum_{i=1}^{n-1} \log \left(\beta^{i}-\beta^{i+1}\right)+\log \left(\beta^{n}\right) \\
& =\frac{n^{2}+n}{2} \log (\beta)+(n-1) \log (1-\beta) .
\end{aligned}
$$

Lemma 16 Given a matrix $S \in \mathbb{R}^{n \times n}$, consider the $n$ dimensional first-order stable spline kernel $K_{\beta}$; then,

$$
\operatorname{Tr}\left\{K_{\beta}^{-1} S\right\}=\sum_{i=1}^{n-1} \frac{d_{i}}{\beta^{i}-\beta^{i+1}}+\frac{d_{n}}{\beta^{n}},
$$

where $d_{i}$ is the ith diagonal element of $\Delta_{n} S \Delta_{n}^{T}$, with $\Delta_{n}$ defined in (37).

PROOF. Follows directly from the factorization (A.7).

Corollary 13 follows directly from Lemma 15 and Lemma 16.

\section{A.5 Proof of Theorem 14}

In the marginal-likelihood problem (16), we construct the complete-data likelihood (25). Using the posterior distribution (10), calculated for some values of the parameters $w=\hat{w}^{(k)}$ and $\rho=\hat{\rho}^{(k)}$, we find the approximation function (A.1).
We now maximize this function with respect to $w$, with $\rho$ fixed at its previous value $\hat{\rho}^{(k)}$, finding $w^{(k+1)}$ (according to Proposition 10). Next, we maximize (A.1) with respect to $\rho$, with $w$ fixed at its updated value $\hat{w}^{(k+1)}$.

To complete the proof, we notice that the maximization steps of the ECM method have the two following properties: the constraint set is space filling (for a complete treatment see [30]), and all the maximizations have unique solutions. In the light of these observations, we can refer to Theorem 1 and Theorem 3 in [30] to conclude that the sequence of updates generated by the ECM method has strictly increasing likelihood and will converge to a stationary point of (16).

\section{References}

[1] B. D. O. Anderson and J. B. Moore. Optimal filtering. Courier Corporation, 2012.

[2] E. W. Bai. An optimal two-stage identification algorithm for Hammerstein-Wiener nonlinear systems. Automatica, 34(3):333-338, 1998.

[3] E. W. Bai, Z. Cai, S. Dudley-Javorosk, and R. K. Shields. Identification of a modified Wiener-Hammerstein system and its application in electrically stimulated paralyzed skeletal muscle modeling. Automatica, 45(3):736-743, 2009.

[4] E. W. Bai and M. Fu. A blind approach to Hammerstein model identification. IEEE Trans. Signal Process., 50(7):1610-1619, 2002.

[5] E. W. Bai and D. Li. Convergence of the iterative Hammerstein system identification algorithm. IEEE Trans. Autom. Control, 49(11):1929-1940, 2004.

[6] S. L. Baumgartner and W. J. Rugh. Complete identification of a class of nonlinear systems from steady-state frequency response. IEEE T. Circuits. Syst., 22(9):753-759, 1975.

[7] S. A. Billings and S. Y. Fakhouri. Identification of a class of nonlinear systems using correlation analysis. Proc. Inst. Electr. Eng., 125(7):691-697, 1978.

[8] C. M. Bishop. Pattern Recognition and Machine Learning. Springer, 2006.

[9] M. Boutayeb, D. Aubry, and M. Darouach. A robust and recursive identification method for MISO Hammerstein model. In Proc. UKACC Int. Conf. Control, volume 1, pages 234-239. IET, 1996.

[10] F. P. Carli. On the maximum entropy property of the firstorder stable spline kernel and its implications. In Proc. IEEE Conf. Control Applicat. (CCA), pages 409-414. IEEE, 2014.

[11] T. Chen and L. Ljung. Constructive state space model induced kernels for regularized system identification. In Proc. IFAC World Cong., volume 19, pages 1047-1052, 2014.

[12] T. Chen, H. Ohlsson, and L. Ljung. On the estimation of transfer functions, regularizations and gaussian processesrevisited. Automatica, 48(8):1525-1535, 2012.

[13] A. Chiuso, G. Pillonetto, and G. De Nicolao. Subspace identification using predictor estimation via Gaussian regression. In Proc. IEEE Conf. Decis. Control (CDC), pages 3299-3304, 2008.

[14] P. Crama and J. Schoukens. Hammerstein-Wiener system estimator initialization. Automatica, 40(9):1543-1550, 2004. 
[15] F. Dinuzzo. Kernels for linear time invariant system identification. SIAM J. Control Optim., 53(5):3299-3317, 2015.

[16] T. Falck, J. A. K. Suykens, J. Schoukens, and B. De Moor. Nuclear norm regularization for overparametrized Hammerstein systems. In Proc. IEEE Conf. Decis. Control (CDC), pages 7202-7207, 2010.

[17] F. Giri and E. W. Bai. Block-oriented nonlinear system identification. Springer, 2010.

[18] I. Goethals, K. Pelckmans, J. A. K. Suykens, and B. De Moor. Subspace identification of Hammerstein systems using least squares support vector machines. IEEE Trans. Autom. Control, 50(10):1509-1519, 2005.

[19] G. Golub and V. Pereyra. Separable nonlinear least squares: the variable projection method and its applications. Inverse Problems, 19(2):R1, 2003.

[20] W. Greblicki. Non-parametric orthogonal series identification of Hammerstein systems. Int. J. Syst. Sci., 20(12):2355-2367, 1989.

[21] W. Greblicki. Continuous-time Hammerstein system identification. IEEE Trans. Autom. Control, 45(6):12321236, 2000.

[22] W. Greblicki and M. Pawlak. Identification of discrete Hammerstein systems using kernel regression estimates. IEEE Trans. Autom. Control, 31(1):74-77, 1986.

[23] Y. Han and R. A. De Callafon. Closed-loop identification of Hammerstein systems using iterative instrumental variables. In Proc. IFAC World Cong., volume 18, pages 13930-13935, 2011.

[24] Y. Han and R. A. De Callafon. Hammerstein system identification using nuclear norm minimization. Automatica, 48(9):2189-2193, 2012.

[25] I. W. Hunter and M. J. Korenberg. The identification of nonlinear biological systems: Wiener and Hammerstein cascade models. Biol. Cybern., 55(2-3):135-144, 1986.

[26] Y. Liu and E. W. Bai. Iterative identification of Hammerstein systems. Automatica, 43(2):346-354, 2007.

[27] L. Ljung. System Identification, Theory for the User. Prentice Hall, 1999.

[28] J. S. Maritz and T. Lwin. Empirical bayes methods. Chapman and Hall London, 1989

[29] G. McLachlan and T. Krishnan. The EM algorithm and extensions, volume 382. John Wiley and Sons, 2007.

[30] X. L. Meng and D. B. Rubin. Maximum likelihood estimation via the ECM algorithm: A general framework. Biometrika, 80(2):267-278, 1993.

[31] G. Mzyk. Generalized kernel regression estimate for the identification of Hammerstein systems. Int. J. Appl. Math. Comput. Sci., 17(2):189-197, 2007.

[32] G. Pillonetto and A. Chiuso. Gaussian processes for WienerHammerstein system identification. In Proc. IFAC Symp. System Identification (SYSID), volume 15, pages 838-843, 2009.

[33] G. Pillonetto and A. Chiuso. Tuning complexity in regularized kernel-based regression and linear system identification: The robustness of the marginal likelihood estimator. Automatica, 58:106-117, 2015.

[34] G. Pillonetto, A. Chiuso, and G. De Nicolao. Prediction error identification of linear systems: a nonparametric gaussian regression approach. Automatica, 47(2):291-305, 2011.
[35] G. Pillonetto and G. De Nicolao. A new kernel-based approach for linear system identification. Automatica, 46(1):81-93, 2010.

[36] G. Pillonetto, F. Dinuzzo, T. Chen, G. De Nicolao, and L. Ljung. Kernel methods in system identification, machine learning and function estimation: A survey. Automatica, 50(3):657-682, 2014.

[37] G. Pillonetto, M. H. Quang, and A. Chiuso. A new kernelbased approach for nonlinear system identification. IEEE Trans. Autom. Control, 56(12):2825-2840, 2011.

[38] R. Pintelon and J. Schoukens. System identification: a frequency domain approach. John Wiley and Sons, 2012.

[39] S. Rangan, G. Wolodkin, and K. Poolla. New results for Hammerstein system identification. In Proc. IEEE Conf. Decis. Control (CDC), volume 1, pages 697-702. IEEE, 1995.

[40] C. E. Rasmussen and C. K. Williams. Gaussian processes for machine learning. the MIT Press, 2006.

[41] R. S. Risuleo, G. Bottegal, and H. Hjalmarsson. A kernelbased approach to Hammerstein system identication. In Proc. IFAC Symp. System Identification (SYSID), volume 48, pages 1011-1016, 2015.

[42] R. S. Risuleo, G. Bottegal, and H. Hjalmarsson. A new kernelbased approach to overparameterized Hammerstein system identification. In Proc. IEEE Conf. Decis. Control (CDC), pages 115-120, 2015.

[43] R. S. Risuleo, G. Bottegal, and H. Hjalmarsson. On the estimation of initial conditions in kernel-based system identification. In Proc. IEEE Conf. Decis. Control (CDC), pages 1120-1125, 2015.

[44] D. Romeres, G. Pillonetto, and A. Chiuso. Identification of stable models via nonparametric prediction error methods. In Proc. European Control Conf. (ECC), pages 2044-2049. IEEE, 2015.

[45] J. Schoukens, T. Dobrowiecki, and R. Pintelon. Parametric and nonparametric identification of linear systems in the presence of nonlinear distortions-a frequency domain approach. IEEE Trans. Autom. Control, 43(2):176-190, 1998.

[46] J. Schoukens, R. Pintelon, T. Dobrowiecki, and Y. Rolain. Identification of linear systems with nonlinear distortions. Automatica, 41(3):491-504, 2005.

[47] J. Sjöberg, Q. Zhang, L. Ljung, A. Benveniste, B. Delyon, P. Y. Glorennec, H. Hjalmarsson, and A. Juditsky. Nonlinear black-box modeling in system identification: a unified overview. Automatica, 31(12):1691-1724, 1995.

[48] L. Vanbeylen, R. Pintelon, and J. Schoukens. Blind maximum-likelihood identification of Wiener systems. IEEE Trans. Signal Process., 57(8):3017-3029, 2009.

[49] M. Verhaegen and D. T. Westwick. Identifying MIMO Hammerstein systems in the context of subspace model identification methods. Int. J. Control, 63(2):331-349, 1996.

[50] G. Wahba. Spline models for observational data, volume 59. SIAM, 1990.

[51] D. T. Westwick and R. E. Kearney. Separable least squares identification of nonlinear Hammerstein models: Application to stretch reflex dynamics. Ann. Biomed. Eng., 29(8):707$718,2001$.

[52] A. Wills, T. B. Schön, L. Ljung, and B. Ninness. Identification of Hammerstein-Wiener models. Automatica, 49(1):70-81, 2013.

[53] A. Yeredor. The joint MAP-ML criterion and its relation to $\mathrm{ML}$ and to extended least-squares. IEEE Trans. Signal Process., 48(12):3484-3492, 2000. 